\title{
New BVRI photometry results on Kuiper Belt Objects from the ESO VLT^
}

\author{
P. Santos-Sanz ${ }^{1}$, J. L. Ortiz ${ }^{1}$, L. Barrera ${ }^{2}$, and H. Boehnhardt ${ }^{3}$ \\ 1 Instituto de Astrofísica de Andalucía, PO Box 3004, 18080 Granada, Spain \\ e-mail: psantos@iaa.es \\ ${ }^{2}$ Universidad Metropolitana de Ciencias de la Educación, Facultad de Ciencias Básicas, Dpto. de Física, Santiago de Chile, Chile \\ 3 Max-Planck-Institute für Solar System Research, Max-Planck-Str. 2, 37191 Katlenburg-Lindau, Germany
}

Received 17 July 2007 / Accepted 26 November 2008

\begin{abstract}
Context. Photometric surveys of transNeptunian objects (TNOs) and Centaurs have suggested possible correlations between some orbital parameters and surface colors of classical objects, scattered disk objects (SDOs), and Centaurs. However, larger sample sizes are needed in order to corroborate or rule out the possible correlations and find some possible new ones. The implications from these correlations for the formation and evolution of classical TNOs, SDOs, and Centaurs are important to understand possible physicochemical coloring mechanisms and their influence on the surfaces of the TNOs and Centaurs, as well as their evolutionary history. Aims. We aim to obtain a sufficiently large photometric dataset in order to carry out a more significant statistical analysis, with emphasis on improving the sample size of observed objects, in particular for classical TNOs and SDOs.

Methods. We use VLT-FORS images through BVRI filters of 32 Kuiper Belt Objects (KBOs) and obtain their colors after proper reduction and calibration. We study the possible correlations (Peixinho et al. 2004, Icarus, 170, 153; Doressoundiram et al. 2005 , Icarus, 174, 90) merging these new measurements with the VLT published results from the ESO large program and with the latest published results of the Meudon Multicolor Survey via non-parametric statistical tests.

Results. We obtain a large dataset of 116 objects (classical, SDOs and Centaurs) and, in addition to confirming most of the correlations and conclusions reached in the literature, some possible new correlations are found. The most interesting ones are some correlations of color vs. orbital parameters for the different dynamical groups. We find that some correlations in the classical group, as well as the (dynamically) cold and hot subgroups depend on the size of the objects. As a by-product of our study, we were able to identify new candidates for light curve studies and found that $\sim 55 \%$ of the objects showed variability above 0.15 mag. This is a higher value than what is found in other studies. Since our sample contains smaller objects than samples from other studies, this result might be an indication that the smaller TNOs are more elongated than the larger ones.
\end{abstract}

Key words. Kuiper Belt - techniques: photometric

\section{Introduction}

Trans-Neptunian objects (TNOs) are cold minor bodies composed of ice and rock that populate the region of the solar system beyond Neptune. Their existence was hypothesized to explain the observed distribution of short-period comets (Fernández 1980; Duncan et al. 1988). The first of these objects, besides Pluto, was discovered in 1992 by Jewitt \& Luu (1993), and currently more than 1000 of these objects have been detected.

Dynamical groups: the known TNOs comprise different dynamical groups: classical objects which exhibit orbits with moderate eccentricities; resonant objects trapped by Neptune in mean motion resonances (the Plutinos being the most representative population, in the 3:2 resonance); scattered disk objects (SDOs) with orbits with high eccentricities and sometimes high inclinations, due to close encounters with the planet Neptune in the past. The classical group is sub-classified in two sub-groups: the so-called (dynamically) hot classical objects with orbital inclinations $>4.5^{\circ}$, and the (dynamically) cold classical objects, with inclinations $<4.5^{\circ}$. Centaurs represent TNOs scattered towards the inner solar system and they reside between the orbits of Jupiter

\footnotetext{
* Based on observations collected at the Very Large Telescope of the European Southern Observatory at Cerro Paranal in Chile under programs 072.C-0483(A) and (B).
}

and Neptune, so many investigators consider them Kuiper Belt Objects (KBOs). The current scenarios of the Solar System formation suggest that the KBOs, the short-period comets and some icy satellites of the major planets were formed in the transNeptunian belt. What is known of their physical nature supports this idea. However, since the TNOs never appear in the vicinity of the Sun, they are believed to be even more pristine than their relatives. The knowledge of the physico-chemical nature of TNOs may give important clues on the conditions prevailing at the time of their formation, mainly for the larger TNOs (some of which comply with the dwarf-planet definition) which are the least collisionally evolved objects.

Colour census of TNOs: over the past years the sample of TNOs with BVRI colors measured has grown to more than 100 objects. Statistical analyses suggest the presence of various correlations among the color and dynamical properties of classical TNOs and SDOs, while Plutinos and Centaurs appear to be heterogeneous and without obvious relations between physical and dynamical parameters of the objects. From BVRI color studies Tegler \& Romanishin (2000) suggested the existence of a group of red classical objects beyond 41 AU. Based upon larger data sets other authors (Boehnhardt et al. 2002; Doressoundiram et al. 2002) provide much clearer evidence for this first TNO group that could be identified from 
similar physical and dynamical properties: classical objects of very red color (spectral gradients above $\sim 30 \% / 100 \mathrm{~nm}$ ) in dynamically cold orbits (low inclinations to the ecliptic and low eccentricities).

Trujillo \& Brown (2002) find a correlation between inclination and surface reddening among classical TNOs and SDOs: the objects tend to be bluer the higher their inclinations. This trend is also shown in the work of Doressoundiram et al. (2002) with the additional emphasis that the high inclination objects are intrinsically brighter (possibly larger) than the dynamically cold ones. Both trends are based on a statistical method that gives higher weight to objects with properties close to the trend line. Consequently, the claimed correlations appear much weaker in normal linear regression fits as performed by Boehnhardt et al. (2002). In addition, they find in the same data set a stronger correlation between the color range of classical objects and perihelion distance: more explicitly, a linear decrease of the spectral gradient with decreasing perihelion distance may exist for classical objects with perihelion between 40 and 36 AU. The correlation coefficients of that trend appear to be higher than those involving inclination. However, at present they are not significant enough for either of the abovementioned correlations to be considered "certain". Moreover, some obvious outliers exist for each of these trends. On the other hand, it appears that the overall spectral gradient range of TNOs (for classicals, SDOs, Plutinos) and Centaurs is confined to -10 and $70 \% / 100 \mathrm{~nm}$ and is fully populated for objects that come closer to the Sun than 35 AU.

Surface evolution scenarios: the standard scenario for the surface evolution of TNOs invokes the counteraction of mainly two effects: (1) chemical alteration due to high-energy radiation and (2) resurfacing by icy deposits after collisions. The first process is expected to produce a surface crust of reddish color, while the latter should cause bluish colors. Some modelling results (Thébault 2003), though using simplified assumptions on the transNeptunian belt structure and the physical resurfacing processes, do not support the abovementioned color trend with inclination. Instead, it predicts a correlation with eccentricity that is not found in the existing data. Gomes (2003) and Morbidelli et al. (2003) introduced an interpretation for the excited classical population (classical objects in orbits of higher inclination and eccentricity, the hot population): these objects could be scattered bodies from the region of the gas giants Neptune and Uranus. Due to the different environment closer to the Sun, these objects may have grown larger before they were injected in the classical region, where they account for the otherwise unexplained hot population of objects. The authors suggest, as a by-product that the chemical properties of the scattered classical objects could be different from those of the cold classicals, which should explain the trend of bluer instead of very red spectral gradients. However, apart from dynamical evidence for the existence of a hot classical population, the arguments for their physical and chemical diversities appear to be of an ad-hoc nature. The color trend with perihelion distance together with further evidence (atmosphere around Pluto, lightcurve change in $1996 \mathrm{TO}_{66}$ ) has led other authors (Hainaut et al. 2000; Boehnhardt et al. 2001, 2002) to propose a third resurfacing effect for TNOs: icy recondensation of gas and deposit of haze from a coma-like atmosphere that is produced by intermittent intrinsic activity of the object. The fresh surface ice would appear bluer. The perihelion, aphelion or semimajor axis dependence could be seen as an indicator of the increasing activity level of the agent driving the activity when the KBOs move closer to the Sun: $\mathrm{N}_{2}$ and CO ice sublimation could be a possibility (Delsemme 1982). Nevertheless, the lightcurve change in $1996 \mathrm{TO}_{66}$ may also be explained as being due to an opposition effect (Belskaya et al. 2006).

The scientific aim of our new observations is that, in order to increase the TNO/Centaur sample size, we present in this paper colors for 32 KBOs. In particular, the SDO and classical sample increase significantly. We merge these measurements with other published results in order to allow a large and unique dataset of objects that we analyse with different statistical methods in order to obtain relevant physical information.

\section{Observations and data reduction}

The observations presented here are a continuation of the Large Program developed for the study of the physical properties of TNOs and Centaurs executed at the European Southern Observatory between 2000 and 2002 (Boehnhardt et al. 2002). Our new observations were performed during 4 nights; 1 st and 2nd November 2003 and 25th and 26th March 2004, with the Very Large Telescope (VLT) unit telescope Antu (8.2 m) at Cerro Paranal, Chile (ESO) using FORS1 focal reducer instrument (detector Tek CCD $2048 \times 2048$ pixels; FOV $=6.8 \times 6.8$ arcsec; resolution $=0.2$ arcsec/pixel). The imaging through Bessell broadband $B V R I$ filters consisted of $R V I R$ exposure sequences of $\sim 1 \mathrm{~h}$ maximum duration to correct for brightness variations due to rotation. Preference was given to fainter KBOs (23-24 mag) in order to compensate the bias towards brighter (most likely larger) objects in the current color sample. For brighter targets we also took $B$ filter photometry (in filter sequences $R B R V I R$ ). In most of the cases the observed objects have well established orbits (2-6 oppositions are measured) except for $2003 \mathrm{QB}_{112}$ that had by then - an observed orbit arc of only 57 days (Table 1 ).

The images were taken under photometric conditions and dark skies. The telescope was tracked at sidereal rate with exposure times between 180-1200 s depending on the object brightness (no differential tracking was needed due to the slow velocity of the KBOs $\sim 1 \operatorname{arcsec} / \mathrm{h}$ ). We choose long enough exposure times to obtain a reasonable signal-to-noise-ratio for the objects $(>25)$. The phase angles of the objects were in the range $0.1-1.2$ deg in order to observe with the optimal object reflectivity position (near opposition) and to avoid possible reddening effects due to larger phase angles (Millis et al. 1976; Bowell \& Lumme 1979).

Data reduction and photometry: each image was reduced using standard techniques of bias subtraction and flat field correction. Landolt standard stars at different airmasses for each night were used in order to calculate the photometric calibration parameters (photometric zero points, first order extinction coefficients and color term corrections).

As the selected TNOs and Centaurs are faint $\left(m_{R} \sim\right.$ 20-24 mag) we measured their magnitudes using small apertures and correcting for the flux loss by means of the aperture correction technique. To carry out this correction, we use 30 unsaturated stars in each image field in order to compute the aperture correction for each photometric object measurement with high accuracy. The object aperture radius varied between 2-6 pixels depending on the night conditions and the brightness of the object. We chose the aperture radius that gave the maximum signal-to-noise-ratio for each object by calculating the curve of growth for a number of stars per image (Howell 1989). An aperture area of $\sim 25$ pixels was found to give the smallest changing correction from image to image. We compute the total error in the photometric process as:

$\sigma=\sqrt{\sigma_{\text {phot }}^{2}+\sigma_{\text {apcorr }}^{2}+\sigma_{\text {calib }}^{2}}$ 
Table 1. Object sample and observational circumstances.

\begin{tabular}{|c|c|c|c|c|c|c|c|}
\hline & Object & Type & UT date & $\Delta(\mathrm{AU})$ & $r(\mathrm{AU})$ & $\alpha(\operatorname{deg})$ & Opps. \\
\hline & $1997 \mathrm{SZ}_{10}$ & $2: 1$ & 2003 Nov. 2 & 31.139 & 32.104 & 0.4 & 3 \\
\hline & $2000 \mathrm{CJ}_{105}$ & Classical(hot) & 2004 Mar. 26 & 46.893 & 47.829 & 0.4 & 4 \\
\hline \multirow[t]{7}{*}{ (60458) } & $2000 \mathrm{CM}_{114}$ & SDO & 2004 Mar. 26 & 42.100 & 42.759 & 1.0 & 4 \\
\hline & $2000 \mathrm{CN}_{105}$ & Classical(cold) & 2004 Mar. 26 & 45.233 & 46.074 & 0.7 & 4 \\
\hline & $2000 \mathrm{CP}_{104}$ & Classical(hot) & 2004 Mar. 25 & 45.651 & 46.499 & 0.7 & 3 \\
\hline & $2000 \mathrm{CR}_{105}$ & ESDO & 2004 Mar. 26 & 53.930 & 54.612 & 0.8 & 3 \\
\hline & $2000 \mathrm{YB}_{2}$ & Classical(cold) & 2003 Nov. 2 & 37.616 & 38.535 & 0.6 & 3 \\
\hline & $2000 \mathrm{YC}_{2}$ & SDO & 2003 Nov. 2 & 38.617 & 39.540 & 0.5 & 3 \\
\hline & $2000 \mathrm{YU}_{1}$ & Classical(hot) & 2003 Nov. 1 & 43.290 & 44.218 & 0.5 & 3 \\
\hline \multirow[t]{10}{*}{ (82075) } & $2000 \mathrm{YW}_{134}$ & SDO & 2004 Mar. 25 & 42.750 & 43.190 & 1.2 & 4 \\
\hline & $2001 \mathrm{FM}_{194}$ & SDO & 2004 Mar. 26 & 35.439 & 36.435 & 0.1 & 3 \\
\hline & $2001 \mathrm{HY}_{65}$ & Classical(hot) & 2004 Mar. 25 & 38.305 & 39.299 & 0.1 & 4 \\
\hline & $2001 \mathrm{HZ}_{58}$ & Classical(cold) & 2004 Mar. 25 & 42.460 & 43.269 & 0.8 & 2 \\
\hline & $2001 \mathrm{QC}_{298}$ & Classical(hot) & 2003 Nov. 1 & 40.051 & 40.563 & 1.2 & 4 \\
\hline & $2001 \mathrm{QO}_{297}$ & Classical(cold) & 2003 Nov. 1 & 42.724 & 43.386 & 1.0 & 3 \\
\hline & $2001 \mathrm{QP}_{297}$ & Classical(cold) & 2003 Nov. 1 & 42.810 & 43.522 & 0.9 & 3 \\
\hline & $2001 Q X_{322}$ & SDO & 2003 Nov. 2 & 38.599 & 39.387 & 0.9 & 2 \\
\hline & $2001 R Z_{143}$ & Classical(cold) & 2003 Nov. 2 & 40.458 & 41.402 & 0.4 & 3 \\
\hline & $2001 S^{73}$ & Cent & 2003 Nov. 2 & 15.021 & 15.999 & 0.6 & 3 \\
\hline \multirow[t]{6}{*}{$(42301)$} & $2^{2001 U_{163}}$ & SDO & 2003 Nov. 2 & 48.313 & 49.264 & 0.3 & 6 \\
\hline & $2002 C_{249}$ & Classical(cold) & 2004 Mar. 25 & 37.743 & 38.699 & 0.4 & 3 \\
\hline & $2002 \mathrm{CX}_{154}$ & SDO & 2004 Mar. 25 & 37.684 & 38.629 & 0.5 & 3 \\
\hline & $2002 \mathrm{CY}_{224}$ & SDO & 2004 Mar. 26 & 35.589 & 36.208 & 1.2 & 3 \\
\hline & $2002 \mathrm{GH}_{32}$ & Classical(hot) & 2004 Mar. 25 & 42.009 & 42.848 & 0.7 & 3 \\
\hline & $2002 \mathrm{GJ}_{32}$ & Classical(hot) & 2004 Mar. 26 & 42.132 & 42.952 & 0.8 & 3 \\
\hline (84522) & $2002 \mathrm{TC}_{302}$ & SDO & 2003 Nov. 2 & 47.080 & 48.055 & 0.2 & 4 \\
\hline \multirow{6}{*}{ (55637) } & $2002 U^{25}$ & Classical(hot) & 2003 Nov. 1 & 41.575 & 42.540 & 0.3 & 5 \\
\hline & $2002 \mathrm{VT}_{130}$ & Classical(cold) & 2003 Nov. 2 & 41.768 & 42.688 & 0.5 & 2 \\
\hline & $2003 \mathrm{AZ}_{84}$ & 3:2(Plutino) & 2003 Nov. 2 & 45.492 & 45.844 & 1.2 & 4 \\
\hline & $2003 Q_{112}$ & Classical(hot) & 2003 Nov. 1 & 38.972 & 39.734 & 0.9 & (57d.) \\
\hline & $2003 Q W_{90}$ & Classical(hot) & 2003 Nov. 1 & 43.718 & 44.484 & 0.8 & 2 \\
\hline & $2003 Q_{111}$ & Classical(cold) & 2003 Nov. 1 & 41.359 & 42.088 & 0.9 & 2 \\
\hline
\end{tabular}

Type: dynamical classification of the objects as described in Sect. 3. $\Delta$ (AU): distance Object-Earth in Astronomical Units. $r$ (AU): distance Object-Sun in Astronomical Units. $\alpha$ (deg): phase angle in degrees. Opps., is the number of observed oppositions of the object for the observation date.

where $\sigma_{\text {phot }}$ is the error of the photometric measurements as estimated from Poisson statistics and using the appropriate CCD parameters.

$\sigma_{\text {apcorr }}$ is the uncertainty of aperture correction resulting from the dispersion among measurements of the field stars. This term includes intrinsically systematic errors like flatfielding gradients and variations.

$\sigma_{\text {calib }}$ is the calibration error. This error is the quadratic sum of the rms of the standard stars fit, and of the derived errors of the zeropoints, extinction coefficients and color-term determinations.

\subsection{Photometry of the new sample}

Overview and comparison with data from other authors: the main color and photometric results of the new sample ordered by the object's designation are listed in Table 2. For the objects that show short-term brightness variability in the $R$-filter (see Table 3 ) we compute the mean value of all $R$-magnitudes of the sequence in order to obtain the color indexes. 13 of the 32 objects had colors measured by other authors: $1997 \mathrm{SZ}_{10}$ (Tegler \& Romanishin 2000); $2000 \mathrm{CM}_{114}, 2000 \mathrm{CR}_{105}, 2001 \mathrm{FM}_{194}, 2001 \mathrm{QX}_{322}, 2001 \mathrm{SQ}_{73}$ (Tegler et al. 2003); 2000CN $105,2000 \mathrm{YW}_{134}$ (Peixinho et al. 2004); 2001UR $163,2002 \mathrm{GH}_{32}, 2002 \mathrm{GJ}_{32}$ (Doressoundiram et al. 2005); 2002 UX 25 (Rabinowitz et al. 2007); and 2003 $\mathrm{AZ}_{84}$ (Fornasier et al. 2004). 5 of these 13 objects show differences in colors and/or magnitudes with respect to our results: $2000 \mathrm{CR}_{105}$, $2001 \mathrm{FM}_{194}, 2001 \mathrm{QX}_{322}, 2001 \mathrm{UR}_{163}$, and $2002 \mathrm{GJ}_{32}$. The differences in colors of $2000 \mathrm{CR}_{105}$ and $2001 \mathrm{QX}_{322}$ are $<2 \sigma$. For $2000 \mathrm{CR}_{105}$ it may be explained by the brightness variability (Table 3). For the other 3 objects the differences in magnitudes or colors are $>2 \sigma$ and may arise from different observational circumstances (different dates, phase angles, etc.), calibration errors, or possible brightness variability due to rotation.

The remaining 19 object measurements are new. Henceforth, "OWN" indicates our own photometric results, "LP" are the VLT published results of the ESO large program (Boehnhardt et al. 2002; Peixinho et al. 2004), and "2MS" are the Meudon Multicolor Survey photometric results (Doressoundiram et al. 2005). We use our own color results in the computations of $\mathrm{OWN}+\mathrm{LP}$ and $\mathrm{OWN}+\mathrm{LP}+2 \mathrm{MS}$ data set in the cases when our objects were repeated measurements in LP, or $2 \mathrm{MS}$.

Color-color diagrams: the color-color plots for our sample are shown in Fig. 1. The top panel shows $V-R$ versus $B-V$. The error bars and different symbols for classical cold objects (open diamonds), classical hot objects (open squares), Neptune's resonant objects (open circles), SDOs (open triangles), Centaur (asterisk) and the Sun (filled triangle).

The reddening line is drawn. This line is the locus of objects displaying a linear reflectivity spectrum and has a range of spectral gradients from -10 to $60 \% / 100 \mathrm{~nm}$ (very red).

There are approximately the same number of points above and below the reddening line. This indicates a roughly 
Table 2. Photometric measurements of our TNO sample.

\begin{tabular}{|c|c|c|c|c|c|c|c|c|}
\hline solar colors & $\begin{array}{c}R \\
(\mathrm{mag})\end{array}$ & $\begin{array}{c}\begin{array}{c}B-V \\
(\mathrm{mag})\end{array} \\
0.67\end{array}$ & $\begin{array}{c}\begin{array}{l}V-R \\
(\mathrm{mag}) \\
0.36\end{array}\end{array}$ & $\begin{array}{c}R-I \\
(\mathrm{mag}) \\
0.33\end{array}$ & $\begin{array}{c}-I \\
(\mathrm{mag}) \\
0.69\end{array}$ & $\begin{array}{c}\begin{array}{c}B-R \\
(\mathrm{mag})\end{array} \\
1.03\end{array}$ & $\begin{array}{c}B-I \\
(\mathrm{mag}) \\
1.36\end{array}$ & $\begin{array}{c}\text { Grt } \\
(\% / 100 \mathrm{~nm})\end{array}$ \\
\hline${ }_{1997 Z_{10}}$ & $23.01 \pm 0.09$ & & $0.85 \pm 0.12$ & $0.57 \pm 0.10$ & $1.42 \pm 0.09$ & & & $43.1 \pm 18.4$ \\
\hline $2000 \mathrm{CJ}_{105}$ & $22.44 \pm 0.04$ & $1.08 \pm 0.08$ & $0.68 \pm 0.07$ & $0.70 \pm 0.06$ & $1.38 \pm 0.07$ & $1.76 \pm 0.07$ & $2.46 \pm 0.06$ & $33.3 \pm 8.1$ \\
\hline$(60458) 2000 \mathrm{CM}_{114}$ & $23.21 \pm 0.04$ & & & $0.66 \pm 0.09$ & $0.75 \pm 0.09$ & & & $16.0 \pm 5.0$ \\
\hline $2000 \mathrm{CN}_{105}$ & $21.72 \pm 0.06$ & $1.06 \pm 0.10$ & $0.66 \pm 0.08$ & $0.69 \pm 0.08$ & $1.35 \pm 0.08$ & $1.72 \pm 0.10$ & $2.41 \pm 0.10$ & $32.0 \pm 10.4$ \\
\hline $2000 \mathrm{CP}_{104}$ & $23.22 \pm 0.03$ & & $0.53 \pm 0.06$ & $0.64 \pm 0.06$ & $1.17 \pm 0.07$ & & & $21.4 \pm 7.3$ \\
\hline $2000 \mathrm{CR}_{105}$ & $23.47 \pm 0.06$ & & $0.28 \pm 0.08$ & $0.87 \pm 0.09$ & $1.16 \pm 0.09$ & & & $15.3 \pm 9.9$ \\
\hline $2000 \mathrm{YB}_{2}$ & $22.24 \pm 0.06$ & $0.86 \pm 0.10$ & $0.64 \pm 0.09$ & $0.55 \pm 0.07$ & $1.18 \pm 0.08$ & $1.49 \pm 0.09$ & $2.04 \pm 0.08$ & $21.2 \pm 9.2$ \\
\hline $2000 \mathrm{YC}_{2}$ & $23.04 \pm 0.05$ & & $0.67 \pm 0.11$ & $0.24 \pm 0.11$ & $0.91 \pm 0.10$ & & & $14.2 \pm 12.5$ \\
\hline $2000 \mathrm{YU}_{1}$ & $22.83 \pm 0.03$ & & $0.66 \pm 0.06$ & $0.52 \pm 0.04$ & $1.19 \pm 0.06$ & & & $25.5 \pm 6.7$ \\
\hline$(82075) 2000 \mathrm{YW}_{134}$ & $20.71 \pm 0.06$ & $1.00 \pm 0.07$ & $0.45 \pm 0.08$ & $0.52 \pm 0.08$ & $0.97 \pm 0.08$ & & & $15.4 \pm 7.4$ \\
\hline $2001 \mathrm{FM}_{194}$ & $22.83 \pm 0.04$ & & $0.47 \pm 0.06$ & $0.62 \pm 0.06$ & $1.09 \pm 0.07$ & & & $16.2 \pm 6.9$ \\
\hline $2001 \mathrm{HY}_{65}$ & $21.93 \pm 0.05$ & $1.03 \pm 0.06$ & $0.48 \pm 0.07$ & $0.71 \pm 0.06$ & $1.19 \pm 0.05$ & $1.51 \pm 0.06$ & $2.22 \pm 0.05$ & $22.6 \pm 6.6$ \\
\hline $2001 \mathrm{HZ}_{58}$ & $22.48 \pm 0.05$ & $1.17 \pm 0.06$ & $0.47 \pm 0.06$ & $0.70 \pm 0.06$ & $1.17 \pm 0.05$ & $1.64 \pm 0.06$ & $2.34 \pm 0.05$ & $24.5 \pm 6.1$ \\
\hline $2001 Q_{2} C_{28}$ & $22.51 \pm 0.05$ & $0.66 \pm 0.07$ & $0.37 \pm 0.07$ & $0.63 \pm 0.07$ & $1.00 \pm 0.06$ & $1.03 \pm 0.08$ & $1.66 \pm 0.06$ & $6.9 \pm 6.7$ \\
\hline $2001 \mathrm{QO}_{297}$ & $22.31 \pm 0.09$ & & $0.69 \pm 0.11$ & $0.58 \pm 0.10$ & $1.27 \pm 0.07$ & & & $30.1 \pm 14.6$ \\
\hline $2001 \mathrm{QP}_{297}$ & $23.03 \pm 0.07$ & & $0.63 \pm 0.10$ & $0.60 \pm 0.09$ & $1.23 \pm 0.10$ & & & $26.6 \pm 12.5$ \\
\hline $2001 Q X_{322}$ & $21.99 \pm 0.06$ & $1.36 \pm 0.13$ & $0.65 \pm 0.11$ & $0.42 \pm 0.09$ & $1.07 \pm 0.11$ & $2.01 \pm 0.11$ & $2.43 \pm 0.11$ & $27.7 \pm 10.4$ \\
\hline $2001 R_{143}$ & $22.36 \pm 0.08$ & $1.08 \pm 0.14$ & $0.51 \pm 0.13$ & $0.49 \pm 0.10$ & $1.00 \pm 0.12$ & $1.59 \pm 0.12$ & $2.07 \pm 0.12$ & $19.0 \pm 11.5$ \\
\hline $2001 \mathrm{SQ}_{73}$ & $20.68 \pm 0.06$ & $0.52 \pm 0.09$ & $0.50 \pm 0.09$ & $0.57 \pm 0.09$ & $1.07 \pm 0.09$ & $1.02 \pm 0.08$ & $1.59 \pm 0.08$ & $6.2 \pm 9.6$ \\
\hline$(42301) 2001 \mathrm{UR}_{163}$ & $20.41 \pm 0.06$ & $1.00 \pm 0.10$ & $1.06 \pm 0.08$ & $0.91 \pm 0.07$ & $1.96 \pm 0.07$ & $2.05 \pm 0.10$ & $2.96 \pm 0.09$ & $68.1 \pm 16.1$ \\
\hline $2002 \mathrm{CC}_{249}$ & $21.87 \pm 0.05$ & & $0.51 \pm 0.08$ & $0.69 \pm 0.06$ & $1.20 \pm 0.07$ & & & $22.3 \pm 8.5$ \\
\hline $2002 \mathrm{CX}_{154}$ & $22.99 \pm 0.05$ & $0.90 \pm 0.10$ & $0.57 \pm 0.08$ & $0.50 \pm 0.07$ & $1.07 \pm 0.08$ & $1.47 \pm 0.09$ & $1.97 \pm 0.09$ & $17.6 \pm 8.2$ \\
\hline $2002 \mathrm{CY}_{224}$ & $21.42 \pm 0.04$ & $1.02 \pm 0.08$ & $0.66 \pm 0.06$ & $0.64 \pm 0.06$ & $1.30 \pm 0.06$ & $1.69 \pm 0.07$ & $2.33 \pm 0.07$ & $29.2 \pm 7.1$ \\
\hline $2002 \mathrm{GH}_{32}$ & $22.35 \pm 0.05$ & $0.99 \pm 0.07$ & $0.57 \pm 0.07$ & $0.59 \pm 0.07$ & $1.16 \pm 0.06$ & $1.56 \pm 0.07$ & $2.15 \pm 0.06$ & $22.3 \pm 7.4$ \\
\hline $2002 \mathrm{GJ}_{32}$ & $21.69 \pm 0.09$ & $1.33 \pm 0.12$ & $0.59 \pm 0.14$ & $0.42 \pm 0.12$ & $1.01 \pm 0.13$ & $1.92 \pm 0.11$ & $2.34 \pm 0.10$ & $24.4 \pm 12.7$ \\
\hline$(84522) 2002 \mathrm{TC}_{302}$ & $20.27 \pm 0.06$ & $1.17 \pm 0.07$ & $0.58 \pm 0.08$ & $0.67 \pm 0.07$ & $1.25 \pm 0.06$ & $1.75 \pm 0.07$ & $2.42 \pm 0.06$ & $28.9 \pm 7.9$ \\
\hline (55637)2002UX ${ }_{25}$ & $19.64 \pm 0.04$ & $0.94 \pm 0.06$ & $0.54 \pm 0.06$ & $0.59 \pm 0.05$ & $1.13 \pm 0.05$ & $1.48 \pm 0.06$ & $2.07 \pm 0.05$ & $19.8 \pm 5.8$ \\
\hline $2002 \mathrm{VT}_{130}$ & $21.68 \pm 0.07$ & $1.45 \pm 0.21$ & $0.56 \pm 0.10$ & $0.63 \pm 0.09$ & $1.19 \pm 0.10$ & $2.00 \pm 0.20$ & $2.63 \pm 0.20$ & $30.8 \pm 11.0$ \\
\hline $2003 \mathrm{AZ}_{84}$ & $20.14 \pm 0.04$ & $0.61 \pm 0.08$ & $0.45 \pm 0.07$ & $0.35 \pm 0.06$ & $0.80 \pm 0.07$ & $1.06 \pm 0.07$ & $1.41 \pm 0.07$ & $1.6 \pm 6.4$ \\
\hline $2003 Q_{112}$ & $22.63 \pm 0.04$ & & $0.75 \pm 0.07$ & $0.01 \pm 0.06$ & $0.77 \pm 0.07$ & & & $12.0 \pm 7.1$ \\
\hline $2003 \mathrm{QW}_{90}$ & $21.17 \pm 0.03$ & $1.11 \pm 0.07$ & $0.67 \pm 0.06$ & $0.65 \pm 0.05$ & $1.32 \pm 0.06$ & $1.78 \pm 0.06$ & $2.43 \pm 0.06$ & $31.5 \pm 6.2$ \\
\hline $2003 Q Y_{111}$ & $23.48 \pm 0.06$ & & $0.46 \pm 0.16$ & $0.69 \pm 0.07$ & $1.15 \pm 0.15$ & & & $18.5 \pm 15.5$ \\
\hline
\end{tabular}

constant spectral slope in the BVR range. One outlier is apparent: $2001 \mathrm{UR}_{163}$ (right open triangle), which is an SDO. This object was measured by Doressoundiram et al. (2005) who obtained redder values than ours. We obtain, nevertheless, that $2001 \mathrm{UR}_{163}$ is a very red object $(B-R=2.05 \pm 0.10)$, not far from the result by Doressoundiram et al. $(B-R=2.28 \pm$ $0.04)$. The difference may be due to rotational color variations, and never due to the opposition effect (because the observing phase angles are the same, $\alpha=0.3$, for both measurements). Nevertheless, Sheppard \& Jewitt (2003) find no measurable photometric variations, with lightcurve amplitude $<0.08 \mathrm{mag}$ and/or period $>24 \mathrm{~h}$. Hence, the different results for this particular object remain unexplained, except if we assume an underestimation of the given errors, perhaps of the calibration ones.

The bottom panel of Fig. 1 shows $R-I$ versus $V-R$, using the same symbols as for the objects in the top panel, now adding the distant TNO $2000 \mathrm{CR}_{105}$ (filled circle) which is considered to be a member of the extended scattered disk. The reddening line is drawn from -10 to $70 \% / 100 \mathrm{~nm}$. The number of points lying above the reddening line is slightly greater than points lying below, which is an indication of a slight increase of the spectral slope over the VRI range. Two outliers are apparent: $2001 \mathrm{UR}_{163}$ (top right open triangle) and $2000 C R_{105}$ (bottom right filled circle). For the color difference of $2001 \mathrm{UR}_{163}$ see the previous paragraph. The color differences of $2000 C R_{105}$ to the overall trend may be explained because this object is a member of the poorly known extended scattered disk population (Gladman et al. 2002) which contains objects with orbits decoupled from Neptune. However, no real evidence for this speculative statement can be provided.

\subsection{Objects showing short-term brightness variability}

From the total sample of 32 objects we have 11 objects with some temporal coverage in the $R$ filter to study a possible shortterm rotational variability. For the analysis described below we take into account objects that show $R$ magnitude variations $\geq 0.15$ mag during the exposure sequence (this value is larger than the mean measurement error listed in Table 2). Among these 11 objects, 7 present short-term variability greater than three times the relative photometric error bars ( $3 \sigma$ variations), and 4 present possible short term variability equal to or below two times the error bars $(\leq 2 \sigma)$ as is shown in Table 3. Here, we use as the error reference $\sigma=\sigma_{\text {phot }}$, i.e. we do not take into account aperture correction $\left(\sigma_{\text {apcorr }}\right)$ or calibration errors $\left(\sigma_{\text {calib }}\right)$, because we observe each object during only one night, and we are computing magnitude variations $(\Delta R \mathrm{~s})$ for small temporal intervals $(\Delta t \mathrm{~s})$ therefore, under these particular conditions, $\sigma_{\text {apcorr }}$ and $\sigma_{\text {calib }}$ are negligible in $\Delta R \mathrm{~s}$.

2 of 11 objects $(\sim 18 \%)$ show variations $\geq 0.40 \mathrm{mag}$, a rate similar to that obtained by Ortiz et al. $(2006, \sim 16 \%)$ and Sheppard \& Jewitt $(2003, \sim 15 \%)$. 4 objects $(\sim 36 \%)$ show variations between 0.15 and $0.40 \mathrm{mag}$, a percentage higher than that by Ortiz et al. $(2006, \sim 15 \%)$ and Sheppard \& Jewitt $(2003, \sim 12 \%)$. In total 6 objects $(\sim 55 \%)$ display variations $\geq 0.15 \mathrm{mag}$, again more than found by Ortiz et al. (2006, 31\%), 
Table 3. Objects with possible short-term brightness variability.

\begin{tabular}{|c|c|c|c|c|c|c|}
\hline & Object & Type & $\Delta t(\min )$ & $\Delta R(\mathrm{mag})$ & $\sigma_{\text {phot }}$ & $R$-range \\
\hline \multirow[t]{7}{*}{$3 \sigma$ var. } & ${ }_{1997 \mathrm{SZ}_{10}}$ & $2: 1$ & 33 & 0.36 & 0.06 & $22.82-23.18$ \\
\hline & $2000 \mathrm{CR}_{105}$ & ESDO & 50 & 0.21 & 0.05 & $23.28-23.49$ \\
\hline & $2000 \mathrm{YC}_{2}$ & SDO & 42 & 0.33 & 0.10 & $22.86-23.19$ \\
\hline & $2000 \mathrm{YU}_{1}$ & Hot & 44 & 0.12 & 0.03 & $23.77-23.89$ \\
\hline & $2003 \mathrm{AZ}_{84}$ & $3: 2$ & 18 & 0.20 & 0.01 & $20.04-20.24$ \\
\hline & $2003 Q_{112}$ & Hot & 151 & 0.48 & 0.05 & $22.40-22.88$ \\
\hline & $2003 Q Y_{111}$ & Cold & 156 & 0.72 & 0.11 & $23.10-23.82$ \\
\hline \multirow[t]{4}{*}{$\leq 2 \sigma$ var. } & $2000 \mathrm{CP}_{104}$ & Hot & 58 & 0.08 & 0.04 & $23.17-23.25$ \\
\hline & $2001 \mathrm{QX}_{322}$ & SDO & 24 & - & 0.05 & $21.97-22.01$ \\
\hline & $2002 U X_{25}$ & Hot & 46 & - & 0.01 & $19.63-19.64$ \\
\hline & $2003 \mathrm{QW}_{90}$ & Hot & 18 & - & 0.01 & $21.16-21.19$ \\
\hline
\end{tabular}

Type, is the dynamical classification of the objects as described in Sect. 3: hot (classical with $i>4.5^{\circ}$ ), cold (classical with $i<4.5^{\circ}$ ), SDO (scattered disk object), ESDO (extended scattered disk object), 2:1 and 3:2 (Neptune's resonant objects). $\Delta t$, is the measurement interval in minutes. $\Delta R$, is the $R$-magnitude variation. $\sigma_{\text {phot }}$ is the uncertainty of the relative photometry of the object (see text). $R$-range, is the total range of the $R$ magnitudes measured. We do not include the $\Delta R$ s that are less than $\sigma_{\text {phot }}$.
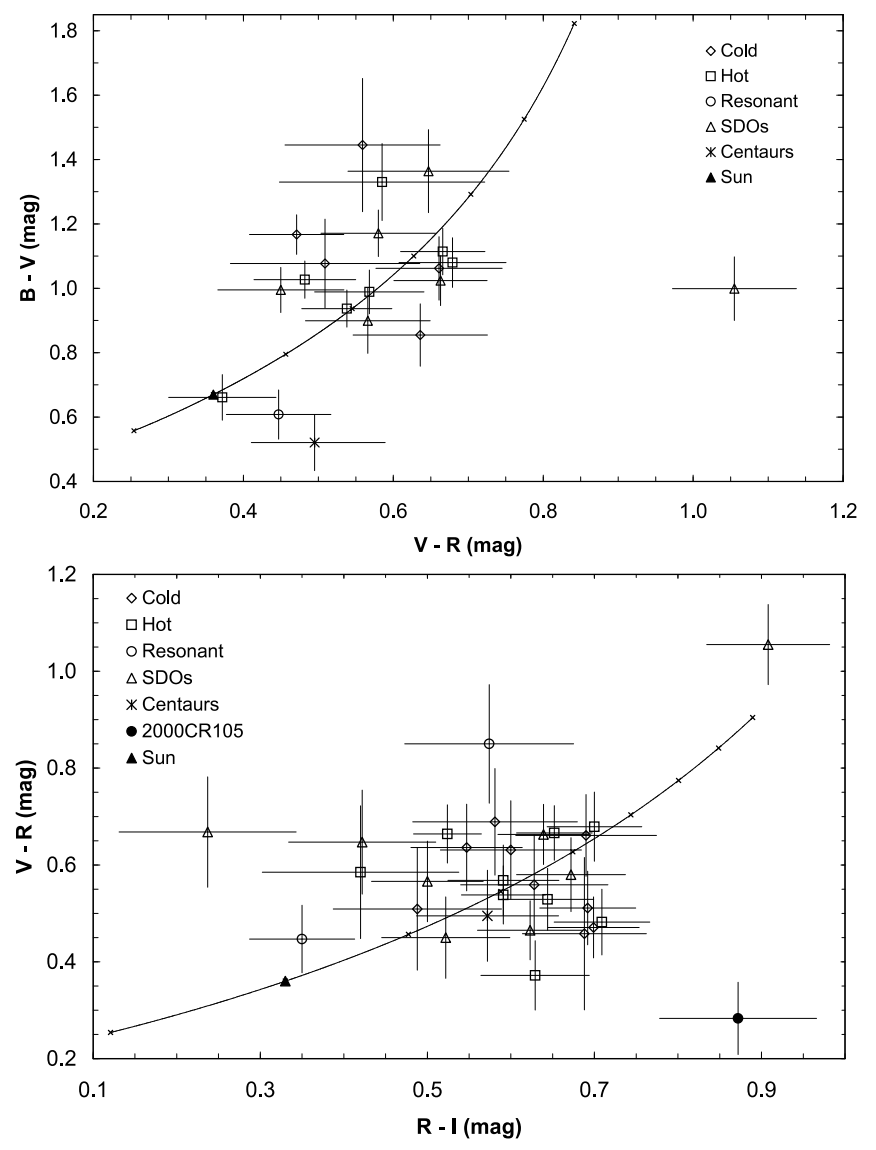

Fig. 1. $V-R$ vs. $B-V$ (top) and $R-I$ vs. $V-R$ (bottom) for the whole new sample. Symbols: classical cold objects (open diamonds), classical hot objects (open squares), Neptune's resonant objects (open circles), SDOs (open triangles), Centaur (asterisk), 2000CR $R_{105}$ (filled circle) and the Sun (filled triangle). The reddening line, which is the locus of objects displaying a linear reflectivity spectrum, has a range of spectral gradients from -10 to $70 \% / 100 \mathrm{~nm}$ (in the top figure only from -10 to $60 \% / 100 \mathrm{~nm}$ ); a cross mark is placed at every 10 units.

Sheppard \& Jewitt (2003, 27\%) and Lacerda \& Luu (2006, $\sim 30 \%$ ). We must take into account too that the average temporal coverage for the eleven objects is $\sim 1 \mathrm{~h}$, a value lower than the typical rotation period for the TNOs (likely to be of the order of a few to several tens of hours). Hence, the amplitude values listed in Table 3 are likely lower limits, which implies that more than $55 \%$ of the objects may have amplitude variations $\geq 0.15 \mathrm{mag}$. This value is higher than those by other authors. However, considering that our objects are significantly fainter than those studied elsewhere, this trend may not be a surprise, because smaller sized objects are expected to be more distorted by collisions than the larger ones, as already pointed out by Lacerda \& Luu (2006). Although less likely there is also the possibility that the photometry errors have been underestimated.

For the colour estimation of the objects that show $R$-brightness variations, we calculate a time interpolated $R$-value for the epoch of the respective complementary filter measurement in the exposure sequence. This interpolated value is the $R$-magnitude shown in Table 2.

\subsection{Absolute magnitudes}

We compute the absolute magnitudes for all the objects using two different methods: the Bowell formalism (Bowell et al. 1989), and the linear phase function approximation. For the Bowell formalism, we use the so-called $G$ parameter. The $G$ value is obtained as an average value weighted with the errors of the $G$ values for TNOs published by Sheppard \& Jewitt (2003). In doing so, we obtain $G=-0.03 \pm 0.02$ for the TNOs. Moreover, we correct the measured magnitude (mag) for the distances object-sun $(r)$ and object-earth $(\Delta)$, to obtain $H_{\alpha}$,

$H_{\alpha}=\operatorname{mag}-5 \cdot \log (r \cdot \Delta)$.

Using the average $G$ value, the phase angle $\alpha$, and the Bowell parameters: $A_{1}=3.33, A_{2}=1.87, B_{1}=0.63, B_{2}=1.22$, we apply the phase correction of the Bowell formalism (Bowell et al. 1989);

$\varphi_{1}=\mathrm{e}^{\left(-A_{1} \cdot \tan (\alpha)^{B_{1}}\right)}$
$\varphi_{2}=\mathrm{e}^{\left(-A_{2} \cdot \tan (\alpha)^{B_{2}}\right)}$.

Then, the absolute magnitude, $H$, is derived as (Bowell et al. 1989):

$H=H_{\alpha}+2.5 \cdot \log \left((1-G) \cdot \varphi_{1}+G \cdot \varphi_{2}\right)$.

On the other hand, we also compute the absolute magnitude using the linear phase function approximation as:

$H=\operatorname{mag}-5 \cdot \log (r \cdot \Delta)-\alpha \cdot \beta$ 
Table 4. Absolute magnitudes for the TNOs of our sample.

\begin{tabular}{|c|c|c|c|c|}
\hline Object & $H_{V}$ (Bowell) & $H_{V}($ Linear $)$ & $H_{R}$ (Bowell) & $H_{R}$ (Linear) \\
\hline $1^{1997 Z_{10}}$ & $8.75 \pm 0.08$ & $8.80 \pm 0.08$ & $7.90 \pm 0.09$ & $7.95 \pm 0.09$ \\
\hline $2000 \mathrm{CJ}_{105}$ & $6.26 \pm 0.06$ & $6.31 \pm 0.06$ & $5.58 \pm 0.05$ & $5.63 \pm 0.04$ \\
\hline $2000 \mathrm{CM}_{114}$ & $6.82 \pm 0.05$ & $6.87 \pm 0.05$ & $6.74 \pm 0.04$ & $6.78 \pm 0.04$ \\
\hline $2000 \mathrm{CN}_{105}$ & $5.63 \pm 0.06$ & $5.68 \pm 0.06$ & $4.97 \pm 0.07$ & $5.02 \pm 0.06$ \\
\hline $2000 \mathrm{CP}_{104}$ & $6.96 \pm 0.06$ & $7.01 \pm 0.06$ & $6.43 \pm 0.03$ & $6.48 \pm 0.03$ \\
\hline $2000 \mathrm{CR}_{105}$ & $6.24 \pm 0.05$ & $6.28 \pm 0.05$ & $5.96 \pm 0.06$ & $6.00 \pm 0.06$ \\
\hline $2000 \mathrm{YB}_{2}$ & $6.93 \pm 0.07$ & $6.98 \pm 0.07$ & $6.30 \pm 0.06$ & $6.34 \pm 0.06$ \\
\hline $2000 \mathrm{YC}_{2}$ & $7.82 \pm 0.08$ & $7.86 \pm 0.07$ & $7.00 \pm 0.05$ & $7.05 \pm 0.05$ \\
\hline $2000 \mathrm{YU}_{1}$ & $6.97 \pm 0.05$ & $7.01 \pm 0.05$ & $6.30 \pm 0.03$ & $6.35 \pm 0.03$ \\
\hline $2000 \mathrm{YW}_{134}$ & $4.61 \pm 0.06$ & $4.65 \pm 0.06$ & $4.16 \pm 0.06$ & $4.20 \pm 0.06$ \\
\hline $2001 \mathrm{FM}_{194}$ & $7.70 \pm 0.05$ & $7.73 \pm 0.05$ & $7.23 \pm 0.04$ & $7.26 \pm 0.04$ \\
\hline $2001 \mathrm{HY}_{65}$ & $6.48 \pm 0.05$ & $6.51 \pm 0.05$ & $6.00 \pm 0.05$ & $6.03 \pm 0.05$ \\
\hline $2001 \mathrm{HZ}_{58}$ & $6.47 \pm 0.05$ & $6.51 \pm 0.04$ & $5.99 \pm 0.05$ & $6.04 \pm 0.05$ \\
\hline $2001 \mathrm{QC}_{298}$ & $6.62 \pm 0.05$ & $6.65 \pm 0.05$ & $6.25 \pm 0.06$ & $6.28 \pm 0.05$ \\
\hline $2001 \mathrm{QO}_{297}$ & $6.47 \pm 0.07$ & $6.51 \pm 0.06$ & $5.78 \pm 0.10$ & $5.82 \pm 0.09$ \\
\hline $2001 \mathrm{QP}_{297}$ & $7.14 \pm 0.08$ & $7.18 \pm 0.08$ & $6.51 \pm 0.07$ & $6.55 \pm 0.07$ \\
\hline $2001 Q X_{322}$ & $6.55 \pm 0.10$ & $6.60 \pm 0.09$ & $5.91 \pm 0.06$ & $5.95 \pm 0.06$ \\
\hline $2001 \mathrm{RZ}_{143}$ & $6.65 \pm 0.10$ & $6.69 \pm 0.10$ & $6.14 \pm 0.08$ & $6.18 \pm 0.08$ \\
\hline $2001 \mathrm{SQ}_{73}$ & $9.14 \pm 0.07$ & $9.21 \pm 0.07$ & $8.64 \pm 0.07$ & $8.71 \pm 0.06$ \\
\hline $2001 \mathrm{UR}_{163}$ & $4.49 \pm 0.06$ & $4.53 \pm 0.06$ & $3.44 \pm 0.06$ & $3.48 \pm 0.06$ \\
\hline $2002 C_{249}$ & $6.46 \pm 0.06$ & $6.50 \pm 0.06$ & $5.95 \pm 0.05$ & $5.99 \pm 0.05$ \\
\hline $2002 \mathrm{CX}_{154}$ & $7.62 \pm 0.07$ & $7.67 \pm 0.07$ & $7.06 \pm 0.05$ & $7.10 \pm 0.05$ \\
\hline $2002 \mathrm{CY}_{224}$ & $6.32 \pm 0.05$ & $6.35 \pm 0.05$ & $5.66 \pm 0.04$ & $5.69 \pm 0.04$ \\
\hline $2002 \mathrm{GH}_{32}$ & $6.49 \pm 0.05$ & $6.54 \pm 0.05$ & $5.92 \pm 0.06$ & $5.97 \pm 0.05$ \\
\hline $2002 \mathrm{GJ}_{32}$ & $5.83 \pm 0.11$ & $5.87 \pm 0.11$ & $5.24 \pm 0.09$ & $5.28 \pm 0.09$ \\
\hline $2002 \mathrm{TC}_{302}$ & $4.01 \pm 0.05$ & $4.05 \pm 0.05$ & $3.43 \pm 0.06$ & $3.47 \pm 0.06$ \\
\hline $2002 U^{25}$ & $3.85 \pm 0.05$ & $3.89 \pm 0.04$ & $3.31 \pm 0.04$ & $3.36 \pm 0.04$ \\
\hline $2002 \mathrm{VT}_{130}$ & $5.87 \pm 0.08$ & $5.91 \pm 0.08$ & $5.31 \pm 0.07$ & $5.35 \pm 0.07$ \\
\hline $2003 \mathrm{AZ}_{84}$ & $3.78 \pm 0.06$ & $3.81 \pm 0.05$ & $3.33 \pm 0.05$ & $3.36 \pm 0.04$ \\
\hline $2003 Q_{112}$ & $7.26 \pm 0.06$ & $7.30 \pm 0.05$ & $6.50 \pm 0.04$ & $6.54 \pm 0.04$ \\
\hline $2003 Q^{90}$ & $5.23 \pm 0.05$ & $5.27 \pm 0.05$ & $4.56 \pm 0.03$ & $4.61 \pm 0.03$ \\
\hline $2003 Q_{111}$ & $7.56 \pm 0.15$ & $7.60 \pm 0.15$ & $7.10 \pm 0.07$ & $7.14 \pm 0.06$ \\
\hline
\end{tabular}

$V$ and $R$ absolute magnitudes for the observed objects estimated using the Bowell formalism $\left(H_{V}(\right.$ Bowell $), H_{R}($ Bowell $\left.)\right)$, and the linear approximation $\left(H_{V}(\right.$ Linear $), H_{R}($ Linear $\left.)\right)$, as is explained in the text.

where mag, $r, \Delta$, and $\alpha$ are the same parameters as explained above and $\beta$ is the phase curve slope or linear phase coefficient. For TNOs we take $\beta=0.16 \pm 0.03 \mathrm{mag} / \mathrm{deg}$, and for Centaurs $\beta=0.11 \pm 0.01 \mathrm{mag} / \mathrm{deg}$ (modal values referring to the $\beta$ estimations by Sheppard \& Jewitt 2002).

For each object we compute the $V$ and $R$ absolute magnitude using the two formalisms above. The four resulting absolute magnitudes ( $H_{V}$ (Bowell), $H_{V}$ (Linear), $H_{R}$ (Bowell), and $H_{R}($ Linear $)$ ) are listed in Table 4 . The absolute magnitudes used for the statistical calculations are given under column $H_{R}$ (Linear), ensuring in this way the compatibility of our data with those by Doressoundiram et al. (2002) and Peixinho et al. (2004).

\subsection{Spectral gradients}

It is known that the TNO and Centaur spectra are approximately featureless in the visible and display almost constant slopes over a wide wavelength range. This is also reflected in the color vs. color plots of our sample (Fig. 1). However, the slope of TNO spectra is not really constant over the full visible spectrum (at least in a certain number of cases); most noteworthy is the slope change towards the near-IR (Davies et al. 2000; Delsanti et al. 2006). Hence, using a set of filters over the full wavelength range for slope fitting can provide quite accurate results, with the possible disadvantage that slopes of some objects are affected by the change-over in the very red, but some others are not affected. In our case we compute the spectral gradients Grt - (the slopes) for the colors $B-V, V-R$ and $R-I$. Taking the average of $\mathrm{Grt}_{(B-V)}, \operatorname{Grt}_{(V-R)}$, and $\mathrm{Grt}_{(R-I)}$, we obtain a single Grt value per object that includes all the color information in the (436, $797 \mathrm{~nm}$ ) wavelength range (Table 2). Hence, first we obtain the partial gradients, $\operatorname{Grt}_{\left(\lambda_{1}-\lambda_{2}\right)}$, using the formula (Hainaut \& Delsanti 2002):

$\operatorname{Grt}_{\left(\lambda_{1}-\lambda_{2}\right)}=\frac{R\left(\lambda_{1}\right)-R\left(\lambda_{2}\right)}{\left|\lambda_{1}-\lambda_{2}\right|} \cdot 10^{4}$

where $\lambda_{1}, \lambda_{2}$ are the central wavelengths of the different filters $(B, V, R$, and $I)$ expressed in nanometers $(\mathrm{nm})$ and $R\left(\lambda_{n}\right)$ are the relative spectral reflectivities (Jewitt \& Meech 1986) normalized to 1 in the $V$-filter central wavelength and expresed as:

$R\left(\lambda_{n}\right)=10^{-0.4 \cdot\left[\left(m\left(\lambda_{n}\right)-m(v)\right)-\left(m\left(\lambda_{n}\right)_{\odot}-m(v)_{\odot}\right)\right]}$

where $m\left(\lambda_{n}\right), m(v)$ are the magnitudes of the object in $\lambda_{n}$ and $V$ filters, $m\left(\lambda_{n}\right)_{\odot}, m(v)_{\odot}$ are the magnitudes of the sun in the same filters. Thereafter, error weighted averaging is performed to obtain the mean value Grt.

The results derived for Grt are given in percent per $100 \mathrm{~nm}$ $(\% / 100 \mathrm{~nm})$. If measurements in all the filters $(B, V, R, I)$ exist, we average the mean value using three partial Grt of adjacent filter colors; if only three filters data are available, the mean value is derived from two partial Grts, etc. The gradient results for our object sample are shown in Table 2 . We consider them to be a good indicator of the gradient slopes over the $B-I$ wavelength 


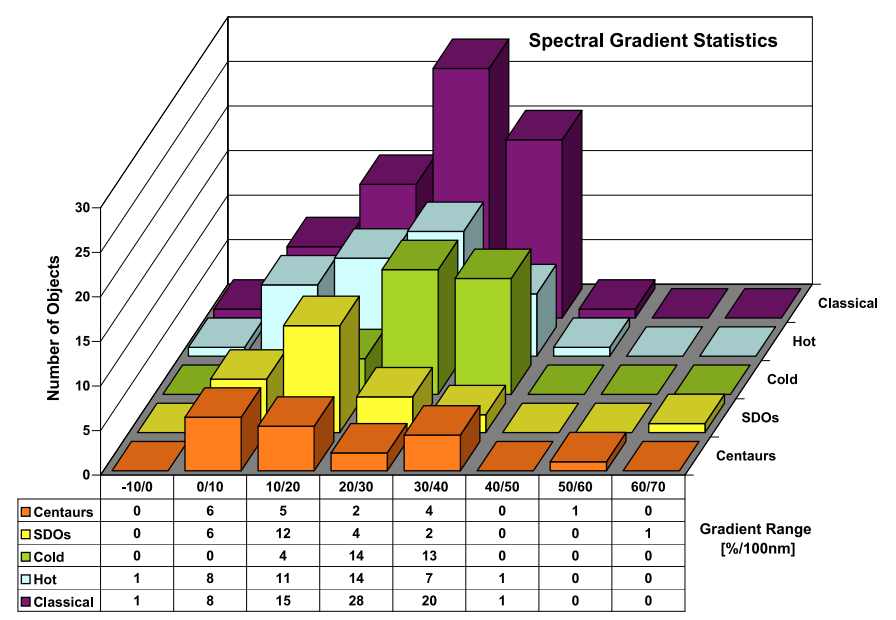

Fig. 2. Reddening gradient statistics of TNOs and Centaurs for the OWN+LP+2MS sample. Dynamical classes: classical, hot, cold, scattered disk objects (SDOs), and Centaurs. The reddening of the objects (in \%/100 nm) as compared to the sun is calculated using Eq. (7) and the number of objects ( $Y$ axis) per reddening interval ( $X$ axis) is counted.

Table 5. Average spectral gradients for TNOs and Centaurs.

\begin{tabular}{ccc}
\hline \hline & $\begin{array}{c}\text { Grt } \\
(\% / 100 \mathrm{~nm})\end{array}$ & $n$ \\
\hline Classical & $22.9 \pm 9.8$ & 73 \\
Hot & $19.6 \pm 8.5$ & 42 \\
Cold & $27.4 \pm 11.3$ & 31 \\
\hline SDOs & $18.6 \pm 7.6$ & 25 \\
\hline Centaurs & $19.8 \pm 6.7$ & 18 \\
\hline
\end{tabular}

Average spectral gradients for the different dynamical groups (except resonant objects) calculated for the whole sample: OWN+LP+2MS. Grt, is the spectral gradient calculated as explained in Sect. 2.4. The errors give the statistical error of the average color and fitted spectral gradient values, respectively. $n$, is the total number of objects used in the average calculation.

range. The gradient errors in the table are computed using the statistical errors of the average colors, and the errors due to the spectral gradient average.

We compute the mean values of spectral gradient and the slope distribution for each dynamical group of TNOs in our own sample using the full datasets: OWN+LP+2MS. We use these results to seek possible correlations of spectral gradients with orbital parameters and $H_{R}$ (see Table 9 below). These results will be discussed in Sect. 3 and subsections thereof. As can be seen in Fig. 2 and in Table 5 there are characteristic differences between the red cold classical cluster, with a high average reddening gradient $(\mathrm{Grt}=27.4 \% / 100 \mathrm{~nm})$, and the hot classical objects, with a low average reddening gradient $(\mathrm{Grt}=19.6 \% / 100 \mathrm{~nm})$, but we can also note that both reddening gradients are compatible within the error bars. Despite possibly different slope distributions, the mean spectral gradients of SDOs and Centaurs agree within errors with that of the hot classicals. Our results are compatible with those obtained by Hainaut \& Delsanti (2002); Boehnhardt et al. (2002, 2003).

\section{Statistical analysis and discussion}

In order to search for correlations between different colors and orbital parameters ( $a$, semimajor axis; $q$, perihelion distance; $Q$, aphelion distance; $e$, eccentricity; $i$, inclination) we use
Table 6. Object summary.

\begin{tabular}{ccccccc}
\hline \hline & & \multicolumn{5}{c}{ Types } \\
& All & Classic & Cold & Hot & SDOs & Cent \\
\hline This work & 30 & 19 & 9 & 10 & $10^{(\star)}$ & 1 \\
LP & 53 & 33 & 16 & 17 & 10 & $10^{(\bullet)}$ \\
2MS & 33 & 21 & 6 & 15 & 5 & 7 \\
\hline Total & 116 & 73 & 31 & 42 & 25 & 18 \\
\hline
\end{tabular}

Number of objects for the different dynamical groups (except the 2 Neptune's resonant objects): All (the whole sample), Classic (classical TNOs), Cold (classical TNOs with inclinations $<4.5^{\circ}$ ), hot (classical TNOs with inclinations $>4.5^{\circ}$ ), SDOs (scattered disk objects), and Cent (Centaurs). LP is the ESO Large Program (Boehnhardt et al. 2002; Peixinho et al. 2004), and 2MS the Meudon Multicolor Survey (Doressoundiram et al. 2005). ${ }^{\star}$ ) We include the peculiar distant object $2000 \mathrm{CR}_{105} \cdot{ }^{(\bullet)}$ We include the uncertain Centaur $2002 \mathrm{CB}_{249}$.

non-parametric statistical tests because these methods do not assume any particular population probability distribution, nor any functional shape. In particular we compute all the correlations using the Spearman rank correlation, $\rho$ (Spearman 1904). This method is distribution-free and less sensitive to outliers than other ones. We studied the strength of the correlations by computing the Spearman coefficient $\rho$ and the significance level (SL) as the probability that the null hypothesis (samples not correlated) is not true. We consider that correlations are strong when $\rho$ is greater than 0.6 , a possible weak correlation exists when $\rho$ values are between 0.3 and 0.6 , and no correlations are present for values less than 0.3. Following Efron \& Tibshirani (1993) we use the qualification criteria: SL greater than $95 \%=$ reasonably strong evidence of correlation; SL greater than $97.5 \%=$ strong evidence of correlation; SL greater than $99 \%=$ very strong evidence of correlation. The interpretation of $\rho$, SL, and the graphical representation of the magnitudes allows us to detect possible correlations among the different magnitudes. Apart from these qualification criteria we consider that a possible correlation could exist when $\rho$ is greater or equal than 0.3 and SL is greater than $80 \%$, in particular for samples with few objects. We emphasize that this particular threshold $(\rho \geq 0.3$; SL $>80 \%)$ is only a hint of a possible correlation that would need more data to be confirmed. First, we correlate only the sample of our new results $(\mathrm{OWN})$, next we merge our objects with the results of the ESO Large Program (LP; Boehnhardt et al. 2002; Peixinho et al. 2004) and, finally, we add the results of the Meudon Multicolor Survey (2MS; Doressoundiram et al. 2005). A summary of the number of objects for each group and sample can be seen in Table 6.

The Spearman statistical analysis of the results is made by separating the dataset in different dynamical subgroups, i.e.: classical objects, hot and cold classical objects, scattered disk objects (SDOs), Centaurs (Cent) etc. We use the classification scheme described in Doressoundiram et al. (2005). Centaurs and SDOs are merged on the MPC list (http://www.cfa. harvard.edu/iau/lists/Centaurs.html) with no distinction. From this list we consider Centaurs the objects with perihelia $(q)$ below 30 AU (Neptune's semi-major axis) and aphelia $(Q)$ below 48 AU (2:1 resonance). The other objects are classified as SDOs ${ }^{1}$. Using the MPC TNOs-list (http://www . cfa.harvard.edu/iau/lists/TNOs.html) we consider as classical objects all non-resonant objects between the 3:2 and

\footnotetext{
1 The only exception is the very distant object $2000 \mathrm{CR}_{105}$ with $Q=$ 394 AU and $a=219$ AU, which is classified as an extended scattered disk object (ESDO).
} 
2:1 resonances $(39.5 \mathrm{AU}<a<48 \mathrm{AU})$ and outside Neptune's Hill sphere $(q>35 \mathrm{AU})$. Objects with low inclined orbits, $q<36 \mathrm{AU}$ and $36 \mathrm{AU}<a<40 \mathrm{AU}$ are also stable and classified as classical objects (Duncan et al. 1995). Two objects are in mean motion resonance with Neptune: $1_{1997 \mathrm{SZ}_{10}}(2: 1$ resonance) and $2003 \mathrm{AZ}_{84}$ (3:2 resonance, Plutino). We do not include these two objects in our statistical analysis. We merge some subsets that could be physically or evolutionally related in order to search for a possible increase in the correlations of the merged sets, i.e.: SDOs+hot, SDOs+Cent. In some cases, color information is not available for some objects (i.e.: in $B$-filter), for this reason we always indicate the number of objects in each correlation computation. The corresponding correlation coefficients and significance levels are listed in Table 7. Here, we discuss only the most relevant results.

\subsection{Color-color correlations}

Correlations of colors are to be expected if one takes for granted the conclusions from measurements of visible spectra of TNOs and Centaurs (see ESO LP papers: Boehnhardt et al. 2002, 2003; Delsanti et al. 2006; Peixinho et al. 2004), i.e. the spectra are generally featureless and have a rather constant slope with only moderate deviations to smaller gradients in the red. Hence, color-color correlations can test the ensemble properties in this respect, i.e. whether or not the general picture of the spectral reddening of the TNOs and Centaurs is consistent for the objects of the sample. In particular, colors covering a wide wavelength range should fulfill the good correlation criteria. Correlations of "neighbouring" colors may be less prominent, since measurement errors can play a greater role. Anti-correlations of colors would be of real interest since they could indicate a unique absorption (less likely emission) feature commonly present in the visible spectra of the ensemble of objects.

The inspection of the correlation parameters for the various object samples, partially included in Table 7, indicates a general agreement with expectations, i.e. good color-color correlations exist for the dynamical classes of classicals (hot and cold ones), SDOs, and Centaurs.

In the following discussion of correlation properties of TNOs and Centaurs, we will not mention explicitly any color-color correlations.

We extended this analysis to the correlation of colors versus the $\psi$ parameter, which is a measure of the average energy of the collisions undergone by a TNO or Centaur. $\psi$ is proportional to the square of the average relative velocity of the object with respect to a circular orbit and depends on the orbital inclination $(i)$, the eccentricity $(e)$, and the semimajor axis $(a)$ through (Opik 1976):

$\psi=\frac{\sin ^{2} i+0.625 \cdot e^{2}}{a}$.

\subsection{All classical objects $(n=73)$}

For the objects of the classical transNeptunian belt we confirm almost all the correlation results and trends found in previous publications (Peixinho et al. 2004; Doressoundiram et al. 2005). However, we are able to identify some new correlations and differences (Table 7). Our own sample of classicals listed in Table 1 amounts to about $26 \%$ of the total number of classicals with colors measured (OWN+LP+2MS).

Strong correlations: the very strong correlations are obtained for colors $B-R$ and $B-V$ vs. inclination $i$ (see Table 7) as well as spectral gradient Grt vs. $i$ (see Table 9). This finding is in agreement with results by other authors (Peixinho et al. 2004; Doressoundiram et al. 2005). We also obtain very strong evidence (SL $>99 \%$ ) for the $B-R$ vs. $q$ and $B-V$ vs. $q$ correlations. Correlations between colors or spectral slopes and the dynamical orbit parameters $i, q$ and $e$ are usually discussed to validate or rule out the collisional resurfacing enviroment (Thebault \& Doressoundiram 2003). Despite the fact that such correlations exist, their interpretation for the surface evolution of the classical TNOs remains unclear since the model predictions are indeed controversial (see Sect. 1 and references therein).

Weaker correlations: here we analyze correlations with low $\rho$ s, but with strong SL values. These correlations are useful for the study because they imply broad but significant trends. Apart from this, we use the threshold $\rho \geq 0.3$ and SL $>80 \%$ as a hint to identify possible correlations, which might be more studied in the future (see Sect. 3). We find three interesting correlations (Tables 7 and 8); $R-I$ vs. $q$, that is not obvious from previous analyses, and two were already known, i.e. $B-R$ vs. $e$, and $V-R$ vs. $q$.

$R-I$ vs. $q$ displays evidence of a very strong correlation $(n=$ $69, \rho=0.30, \mathrm{SL}=98.71 \%$ ) which supports the scenario that the redder objects have orbits with larger perihelion distance. This is consistent with the already known strong $B-R$ vs. $q$ correlation.

Regarding $B-R$ vs. $e, i$, and $q$ we can say that the classicals present (1) higher inclinations the bluer they are; (2) shorter perihelion distances and higher eccentricity. These objects are usually referred to as the dynamically hot population. Vice versa, the red classicals have lower inclinations, greater perihelion distances and lower eccentricity orbits; they represent the dynamically cold population. The short perihelion distances of the bluer objects could support a coloring scenario by resurfacing with fresh "bluish" ices due to sublimation of rare volatile species (Delsemme 1982) around perihelion that freeze out subsequently when the object is further from the Sun. Other coloring mechanisms may explain these correlations as well, such as micrometeoroid bombardment, that would operate preferentially at smaller perihelion distances, where the particle number density might be higher.

Dependences on "sizes": we also searched for possible dependences of the correlations on object "sizes", which is equivalent to a dependence on absolute magnitudes $\left(H_{R}\right)$ if one assumes the same albedo for the objects. This should be a reasonable assumption because we analyze here only faint classical objects, which are expected to be a quite homogeneous sample. We obtain an average $R$-albedo $\left(p_{R}=0.12\right)$ from 28 measured albedos of classical TNOs compiled from different authors (Brown \& Trujillo 2004; Cruiksahnk et al. 2005; Grundy et al. 2005; Jewitt et al. 2001; Lykawka \& Mukai 2005; Margot et al. 2002; Noll et al. 2004; Osip et al. 2003; Sheppard \& Jewitt 2002; Stansberry et al. 2005, 2008). One can speculate on such a dependence on sizes, since the smaller objects are probably collisionally more evolved. For this reason the largest objects should present different physico-chemical properties than the smallest ones. Peixinho et al. (2004) studied the color-perihelion trend for the classical objects, in particular for the $B-R$ vs. $q$ correlation. Using this cut-off brightness of $H_{R}<6.2 \mathrm{mag}$ as a proxy for a minimum size of the objects (diameter $D>190 \mathrm{~km}$ for an albedo $p_{R}=0.12$ ), they find a significant increase of the correlation of the $B-R$ color values with perihelion distance $q$ for classical objects, and a decrease of the correlation when $H_{R}>6.2(D<190 \mathrm{~km})$. Objects with shorter perihelion distance are closer to the Sun and would be susceptible to develop some sort of bound coma if they are large enough $(D>150 \mathrm{~km})$ 
Table 7. Correlations.

\begin{tabular}{|c|c|c|c|c|c|c|c|c|c|c|}
\hline & \multirow[b]{2}{*}{ correlated magnitudes } & \multicolumn{3}{|c|}{ OWN } & \multicolumn{3}{|c|}{ OWN+LP } & \multicolumn{3}{|c|}{$\mathrm{OWN}+\mathrm{LP}+2 \mathrm{MS}$} \\
\hline & & $\rho$ & SL(\%) & $n$ & $\rho$ & $\operatorname{SL}(\%)$ & $n$ & $\rho$ & SL(\%) & $n$ \\
\hline \multirow[t]{7}{*}{ Classicals } & $B-V$ vs. $R-I$ & 0.05 & 15.66 & 12 & 0.40 & 99.03 & 42 & 0.34 & 99.09 & 61 \\
\hline & $R-I$ vs. $B-R$ & 0.03 & 8.33 & 12 & 0.50 & 99.87 & 42 & 0.38 & 99.70 & 61 \\
\hline & $B-V$ vs. $q$ & 0.59 & 95.13 & 12 & 0.37 & 98.24 & 43 & 0.40 & 99.83 & 64 \\
\hline & $B-R$ vs. $q$ & 0.55 & 92.96 & 12 & 0.46 & 99.71 & 43 & 0.46 & 99.98 & 64 \\
\hline & $B-V$ vs. $i$ & -0.63 & 96.24 & 12 & -0.48 & 99.83 & 43 & -0.49 & 99.99 & 64 \\
\hline & $B-R$ vs. $i$ & -0.57 & 94.32 & 12 & -0.54 & 99.96 & 43 & -0.54 & $>99.99$ & 64 \\
\hline & $B-R v s . e$ & -0.38 & 79.79 & 12 & -0.31 & 95.28 & 43 & -0.29 & 98.06 & 64 \\
\hline \multirow[t]{11}{*}{ Hot } & $B-V$ vs. $V-R$ & 0.75 & 93.38 & 7 & 0.78 & 99.97 & 23 & 0.56 & 99.94 & 38 \\
\hline & $B-V$ vs. $R-I$ & -0.02 & 3.52 & 7 & 0.49 & 97.83 & 23 & 0.41 & 98.38 & 36 \\
\hline & $V-R$ vs. $R-I$ & -0.32 & 67.53 & 10 & 0.39 & 95.27 & 27 & 0.29 & 92.79 & 40 \\
\hline & $R-I$ vs. $B-R$ & -0.18 & 34.11 & 7 & 0.53 & 98.76 & 23 & 0.41 & 98.50 & 36 \\
\hline & $B-V$ vs. $q$ & 0.29 & 51.60 & 7 & 0.41 & 94.71 & 23 & 0.38 & 97.96 & 38 \\
\hline & $V-R$ vs. $q$ & 0.18 & 40.20 & 10 & 0.29 & 86.08 & 27 & 0.46 & 99.67 & 42 \\
\hline & $B-R$ vs. $q$ & 0.32 & 56.89 & 7 & 0.36 & 91.02 & 23 & 0.49 & 99.68 & 38 \\
\hline & $B-V$ vs. $i$ & -0.90 & 97.27 & 7 & -0.72 & 99.93 & 23 & -0.47 & 99.57 & 38 \\
\hline & $V-R$ vs. $i$ & -0.51 & 87.45 & 10 & -0.58 & 99.70 & 27 & -0.40 & 98.98 & 42 \\
\hline & $B-R$ vs. $i$ & -0.82 & 95.77 & 7 & -0.64 & 99.72 & 23 & -0.48 & 99.67 & 38 \\
\hline & $B-V v s . e$ & -0.50 & 77.93 & 7 & -0.34 & 89.33 & 23 & -0.25 & 86.82 & 38 \\
\hline \multirow[t]{2}{*}{ Cold } & $B-V$ vs. $V-R$ & -0.60 & 76.99 & 5 & -0.64 & 99.50 & 20 & -0.39 & 94.91 & 26 \\
\hline & $V-R$ vs. $R-I$ & -0.33 & 65.42 & 9 & -0.30 & 83.42 & 23 & -0.38 & 95.41 & 29 \\
\hline \multirow[t]{10}{*}{ SDOs } & $B-V$ vs. $V-R$ & 0.43 & 66.21 & 6 & 0.63 & 97.14 & 13 & 0.76 & 99.87 & 19 \\
\hline & $R-I$ vs. $B-R$ & 0.37 & 59.38 & 6 & 0.30 & 70.11 & 13 & 0.62 & 99.19 & 19 \\
\hline & $B-V$ vs. $q$ & -0.54 & 77.52 & 6 & 0.70 & 98.44 & 13 & 0.36 & 87.66 & 19 \\
\hline & $R-I v s . q$ & 0.17 & 36.26 & 9 & 0.35 & 86.46 & 19 & 0.21 & 69.30 & 25 \\
\hline & $B-R$ vs. $q$ & -0.60 & 82.03 & 6 & 0.62 & 96.85 & 13 & 0.34 & 85.54 & 19 \\
\hline & $B-V v s . i$ & 0.49 & 72.26 & 6 & 0.49 & 90.97 & 13 & 0.29 & 77.80 & 19 \\
\hline & $B-V$ vs. $e$ & 0.03 & 5.09 & 6 & -0.59 & 95.83 & 13 & -0.32 & 81.97 & 19 \\
\hline & $R-I$ vs. $e$ & -0.62 & 91.89 & 9 & -0.46 & 95.11 & 19 & -0.31 & 86.93 & 25 \\
\hline & $B-V$ vs. $H_{R}$ & -0.26 & 43.47 & 6 & -0.77 & 99.27 & 13 & -0.54 & 97.73 & 19 \\
\hline & $B-R$ vs. $H_{R}$ & -0.31 & 51.78 & 6 & -0.81 & 99.49 & 13 & -0.57 & 98.41 & 19 \\
\hline \multirow[t]{10}{*}{ Cent } & $B-V$ vs. $V-R$ & & & & 0.55 & 88.02 & 9 & 0.67 & 99.07 & 16 \\
\hline & $B-V$ vs. $R-I$ & & & & 0.58 & 90.10 & 9 & 0.71 & 99.43 & 16 \\
\hline & $V-R$ vs. $Q$ & & & & 0.65 & 96.15 & 11 & 0.58 & 98.37 & 18 \\
\hline & $B-V$ vs. $i$ & & & & -0.62 & 91.89 & 9 & -0.53 & 95.97 & 16 \\
\hline & $B-R$ vs. $i$ & & & & -0.68 & 94.67 & 9 & -0.52 & 95.39 & 16 \\
\hline & $B-V$ vs. $e$ & & & & 0.72 & 95.73 & 9 & 0.74 & 99.59 & 16 \\
\hline & $B-R$ vs. $e$ & & & & 0.58 & 90.10 & 9 & 0.67 & 99.08 & 16 \\
\hline & $V-R$ vs. $a$ & & & & 0.61 & 94.59 & 11 & 0.53 & 97.04 & 18 \\
\hline & $B-V$ vs. $H_{R}$ & & & & -0.72 & 95.73 & 9 & -0.18 & 50.57 & 16 \\
\hline & $B-R$ vs. $H_{R}$ & & & & -0.73 & 96.19 & 9 & -0.27 & 70.83 & 16 \\
\hline \multirow[t]{3}{*}{ All } & $B-V$ vs. $V-R$ & 0.29 & 78.06 & 19 & 0.50 & 99.99 & 65 & 0.55 & $>99.99$ & 99 \\
\hline & $B-V$ vs. $R-I$ & 0.07 & 24.84 & 19 & 0.46 & 99.98 & 64 & 0.49 & $>99.99$ & 96 \\
\hline & $R-I$ vs. $B-R$ & 0.23 & 66.51 & 19 & 0.56 & $>99.99$ & 64 & 0.54 & $>99.99$ & 96 \\
\hline \multirow[t]{4}{*}{ SDOs+hot } & $B-V$ vs. $V-R$ & 0.55 & 94.30 & 13 & 0.70 & $>99.99$ & 36 & 0.62 & $>99.99$ & 57 \\
\hline & $B-V$ vs. $R-I$ & $<0.01$ & 2.28 & 13 & 0.44 & 99.01 & 36 & 0.47 & 99.95 & 55 \\
\hline & $R-I$ vS. $B-R$ & 0.17 & 44.54 & 13 & 0.51 & 99.76 & 36 & 0.52 & 99.99 & 55 \\
\hline & $V-R$ vs. $i$ & -0.55 & 97.72 & 18 & -0.44 & 99.60 & 44 & -0.35 & 99.44 & 65 \\
\hline \multirow[t]{4}{*}{ SDOs+Cent } & $B-V$ vs. $V-R$ & 0.57 & 83.84 & 7 & 0.66 & 99.73 & 22 & 0.73 & $>99.99$ & 35 \\
\hline & $B-V$ vs. $R-I$ & 0.04 & 6.97 & 7 & 0.47 & 96.88 & 22 & 0.64 & 99.98 & 35 \\
\hline & $V-R$ vs. $R-I$ & 0.13 & 29.39 & 9 & 0.34 & 92.27 & 28 & 0.46 & 99.64 & 41 \\
\hline & $R-I$ vs. $B-R$ & 0.32 & 56.89 & 7 & 0.48 & 97.18 & 22 & 0.63 & 99.98 & 35 \\
\hline
\end{tabular}

Spearman correlation results of colors versus colors and orbital parameters. OWN means our own data; OWN+LP our own data merged with the ESO Large Program data (Boehnhardt et al. 2002; Peixinho et al. 2004); OWN+LP+2MS the OWN+LP data merged with the Meudon Multicolor Survey data (Doressoundiram et al. 2005), $\rho=$ Spearman rank correlation, SL $=$ Significance level, $n=$ Number of objects. Strongest correlations are in bold; interesting or new, but weaker correlations are in italics.

(Delsanti et al. 2004). Their surfaces may experience deposition of fresh bluish ices when the object moves further away from perihelion, so that the objects would appear bluer than before.

We find a slight increase in the $B-R$ vs. $q$ correlation for the classicals $(\mathrm{OWN}+\mathrm{LP}+2 \mathrm{MS})$ with $H_{R}<6.2$ (Table 8), but with a not fully convincing level of significance (SL). Similar results are obtained for $B-V$ vs. $q$. Hence, we cannot conclude on firm color vs. perihelion distance dependence on size of the classical objects. Neither do we find dependence on sizes for the spectral gradients versus $q$. It is noteworthy, however, that opposite trends may exist for $R-I$ vs. $q$ in the sense that it increases for $H_{R}>6.2$, and decreases for $H_{R}<6.2$ (Table 8). This result 
Table 8. Correlations and dependence on "sizes".

\begin{tabular}{|c|c|c|c|c|c|c|c|c|c|c|}
\hline & & & OWN & & & $\mathrm{DWN}+\mathrm{L}$ & & & $\mathrm{N}+\mathrm{LP}+$ & \\
\hline & correlated magnitudes & $\rho$ & $\mathrm{SL}(\%)$ & $n$ & $\rho$ & $\operatorname{SL}(\%)$ & $n$ & $\rho$ & $\operatorname{SL}(\%)$ & $n$ \\
\hline Classicals & $B-V$ vs. $q$ & 0.59 & 95.13 & 12 & 0.37 & 98.24 & 43 & 0.40 & 99.83 & 64 \\
\hline & $B-V$ vs. $q\left(H_{R}>6.2\right)$ & & & 2 & 0.28 & 84.33 & 27 & 0.33 & 93.04 & 32 \\
\hline & $B-V$ vs. $q\left(H_{R}<6.2\right)$ & 0.64 & 94.37 & 10 & 0.52 & 95.50 & 16 & 0.49 & 99.40 & 32 \\
\hline & $R-I$ vs. $q$ & 0.17 & 52.53 & 19 & 0.32 & 97.63 & 50 & 0.30 & 98.71 & 69 \\
\hline & $R-I$ vs. $q\left(H_{R}>6.2\right)$ & 0.71 & 91.98 & 7 & 0.57 & 99.85 & 31 & 0.51 & 99.68 & 35 \\
\hline & $R-I$ vs. $q\left(H_{R}<6.2\right)$ & -0.10 & 26.38 & 12 & -0.09 & 30.42 & 19 & 0.06 & 26.99 & 34 \\
\hline & $B-R$ vs. $q$ & 0.55 & 92.96 & 12 & 0.46 & 99.71 & 43 & 0.46 & 99.98 & 64 \\
\hline & $B-R$ vs. $q\left(H_{R}>6.2\right)$ & & & 2 & 0.48 & 98.58 & 27 & 0.47 & 99.06 & 32 \\
\hline & $B-R$ vs. $q\left(H_{R}<6.2\right)$ & 0.55 & 90.20 & 10 & 0.43 & 90.37 & 16 & 0.49 & 99.39 & 32 \\
\hline & $R-I$ vs. $e$ & 0.17 & 54.82 & 19 & -0.21 & 86.62 & 50 & -0.17 & 84.83 & 69 \\
\hline & $R-I$ vs. $e\left(H_{R}>6.2\right)$ & 0.14 & 27.36 & 7 & -0.41 & 97.68 & 31 & -0.38 & 97.28 & 35 \\
\hline & $R-I$ vs. $e\left(H_{R}<6.2\right)$ & 0.15 & 38.26 & 12 & 0.13 & 42.35 & 19 & 0.04 & 19.76 & 34 \\
\hline & $V-R$ vs. $a$ & -0.14 & $\overline{45.84}$ & 19 & 0.18 & 80.79 & 52 & 0.12 & 69.48 & 73 \\
\hline & $V-R$ vs. $a\left(H_{R}>6.2\right)$ & -0.43 & 70.62 & 7 & -0.06 & 26.64 & 33 & -0.02 & 10.12 & 38 \\
\hline & $V-R$ vs. $a\left(H_{R}<6.2\right)$ & 0.03 & 7.39 & 12 & 0.48 & 96.01 & 19 & 0.21 & 76.84 & 35 \\
\hline & $B-R$ vs. $a$ & 0.08 & 21.92 & 12 & 0.09 & 43.80 & 43 & 0.14 & 73.85 & 64 \\
\hline & $B-R$ vs. $a\left(H_{R}>6.2\right)$ & & & 2 & -0.19 & 67.17 & 27 & -0.11 & 44.33 & 32 \\
\hline & $B-R$ vs. $a\left(H_{R}<6.2\right)$ & 0.25 & 54.40 & 10 & 0.56 & 97.04 & 16 & 0.29 & 89.64 & 32 \\
\hline & $V-R$ vs. $\psi$ & 0.20 & 61.21 & 19 & 0.05 & 27.68 & 52 & 0.05 & 30.00 & 73 \\
\hline & $V-R$ vs. $\psi\left(H_{R}>6.2\right)$ & 0.14 & 27.36 & 7 & 0.28 & 88.87 & 33 & 0.35 & 96.69 & 38 \\
\hline & $V-R$ vs. $\psi\left(H_{R}<6.2\right)$ & 0.10 & 25.46 & 12 & -0.34 & 84.70 & 19 & -0.28 & 89.76 & 35 \\
\hline & $B-R$ vs. $\psi$ & 0.20 & 48.39 & 12 & $<0.01$ & 1.44 & 43 & -0.02 & 14.42 & 64 \\
\hline & $B-R$ vs. $\psi\left(H_{R}>6.2\right)$ & & & 2 & 0.06 & 24.44 & 27 & 0.19 & 70.87 & 32 \\
\hline & $B-R$ vs. $\psi\left(H_{R}<6.2\right)$ & -0.07 & 15.85 & 10 & -0.23 & 62.57 & 16 & -0.32 & 92.06 & 32 \\
\hline Hot & $B-V$ vs. $q$ & 0.29 & 51.60 & 7 & 0.41 & 94.71 & 23 & 0.38 & 97.96 & 38 \\
\hline & $B-V$ vs. $q\left(H_{R}>6.2\right)$ & & & 1 & 0.20 & 51.87 & 13 & 0.31 & 78.33 & 17 \\
\hline & $B-V$ vs. $q\left(H_{R}<6.2\right)$ & 0.77 & 91.55 & 6 & 0.70 & 96.35 & 10 & 0.39 & 91.46 & 21 \\
\hline & $R-I$ vs. $q$ & 0.20 & 45.27 & 10 & 0.23 & 75.89 & 27 & 0.19 & 76.46 & 40 \\
\hline & $R-I$ vs. $q\left(H_{R}>6.2\right)$ & 0.60 & 70.13 & 4 & 0.37 & 84.55 & 16 & 0.20 & 61.21 & 19 \\
\hline & $R-I$ vs. $q\left(H_{R}<6.2\right)$ & 0.09 & 15.42 & 6 & $<0.01$ & 2.30 & 11 & 0.13 & 45.04 & 21 \\
\hline & $V-R$ vs. $a$ & -0.31 & 64.62 & 10 & 0.19 & 65.45 & 27 & 0.21 & 82.91 & 42 \\
\hline & $V-R$ vs. $a\left(H_{R}>6.2\right)$ & & & 4 & -0.23 & 61.99 & 16 & -0.16 & 51.09 & 20 \\
\hline & $V-R$ vs. $a\left(H_{R}<6.2\right)$ & 0.37 & 59.38 & 6 & 0.67 & 96.66 & 11 & 0.49 & 97.41 & 22 \\
\hline & $B-R$ vs. $a$ & $<0.01$ & $<0.01$ & 7 & 0.12 & 41.24 & 23 & 0.22 & 82.46 & 38 \\
\hline & $B-R$ vs. $a\left(H_{R}>6.2\right)$ & & & 1 & -0.13 & 33.84 & 13 & -0.09 & 26.85 & 17 \\
\hline & $B-R$ vs. $a\left(H_{R}<6.2\right)$ & 0.60 & 82.03 & 6 & 0.79 & 98.28 & 10 & 0.59 & 99.15 & 21 \\
\hline Cold & $R-I$ vs. $q$ & 0.15 & 32.86 & 9 & 0.25 & 75.53 & 23 & 0.27 & 84.35 & 29 \\
\hline & $R-I$ vs. $q\left(H_{R}>6.2\right)$ & & & 3 & 0.56 & 96.29 & 15 & 0.60 & 98.06 & 16 \\
\hline & $R-I$ vs. $q\left(H_{R}<6.2\right)$ & -0.14 & 25.06 & 6 & -0.45 & 76.86 & 8 & -0.30 & 69.60 & 13 \\
\hline & $V-R$ vs. $a$ & -0.17 & 36.26 & 9 & 0.35 & 91.29 & 25 & 0.09 & 38.31 & 31 \\
\hline & $V-R$ vs. $a\left(H_{R}>6.2\right)$ & -0.50 & 52.05 & 3 & 0.41 & 90.05 & 17 & 0.42 & 91.52 & 18 \\
\hline & $V-R$ vs. $a\left(H_{R}<6.2\right)$ & -0.20 & 34.53 & 6 & 0.17 & 34.08 & 8 & -0.30 & 69.60 & 13 \\
\hline
\end{tabular}

Correlations results of colors versus orbital parameters and versus $\psi$, a measure of the "average Collisional Energy" (Opik 1976) for classical objects. We correlate the whole group and two different subgroups of different "sizes" with $H_{R}>6.2$, and $H_{R}<6.2$. OWN means our own data, OWN+LP means our own data merged with the ESO Large Program data (Boehnhardt et al. 2002; Peixinho et al. 2004), OWN+LP+2MS means the OWN+LP data merged with the Meudon Multicolor Survey data (Doressoundiram et al. 2005), $\rho=$ Spearman rank correlation, $\mathrm{SL}=$ Significance level, $n=$ Number of objects.

could imply that $R-I$ correlates best with perihelion distance for the smaller classical objects, i.e. the smaller classical objects are bluer (less red) for the lower perihelion distances $(q)$. This $q$-dependent coloring mechanism cannot be due to like-comet activity, because these objects are very small to retain a coma. Micrometeoroid bombardment or other $q$-dependence coloring mechanism might be necessary to explain this particular trend. This finding should be taken with care since it affects only a single color and thus requires a very careful physical interpretation that is currently not available. We do not find size dependencies versus aphelion distance $(Q)$.

We find a possible dependence on sizes of the color correlations with semimajor axis $(a)$, in particular for $B-R$ vs. $a$
$\left(H_{R}<6.2\right)$ for the OWN+LP sample $(\rho=0.56, \mathrm{SL}=97.04 \%)$. A similar trend is apparent for $V-R$ vs. $a(\mathrm{OWN}+\mathrm{LP})$ and $R-I$ vs. $a(\mathrm{OWN}+\mathrm{LP}+2 \mathrm{MS})$ as well. We reproduce this finding in the $B-R$ vs. $\psi\left(H_{R}<6.2\right)$ correlation (Table 8), however as a possible weak anti-correlation $(\rho=-0.32, \mathrm{SL}=92.06 \%$, $n=32$ ). These results are compatible since $\psi$ depends on $\frac{1}{a}$. They are also supported by our findings for Grt vs. $\psi\left(H_{R}<6.2\right)$, see Table 9.

Thus for the larger classical objects $(D>190 \mathrm{~km})$ there is an apparent trend of increased reddening with larger semimajor axis or, in other words, they appear bluer the closer their mean distances to the Sun are. Based on this finding, one may speculate that these bodies are large enough to retain volatiles 
Table 9. Remarkable or interesting correlations of computed spectral gradients.

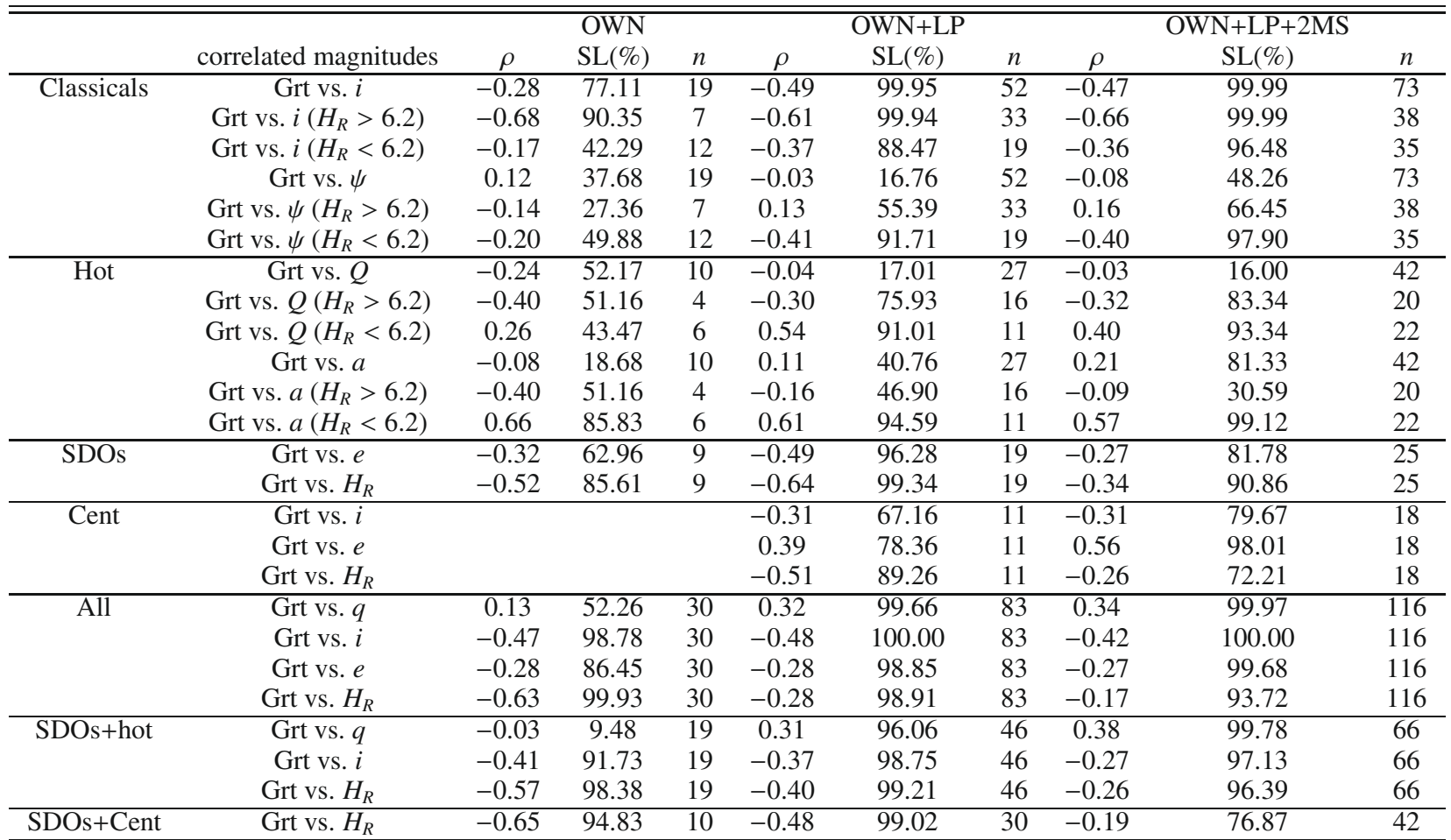

Interesting correlations of the spectral gradient (Grt) versus $H_{R}$ and orbital parameters discussed in text. The spectral gradient, Grt(\%/100 nm), was calculated by fitting over the available color intervals per object. OWN means our own data, OWN+LP means our own data merged with the ESO Large Program data (Boehnhardt et al. 2002; Peixinho et al. 2004), OWN+LP+2MS means the OWN+LP data merged with the Meudon Multicolor Survey data (Doressoundiram et al. 2005), $\rho=$ Spearman rank correlation, $\mathrm{SL}=$ Significance level, $n=$ Number of objects .

produced either by impacts or intrinsic activity. Subsequent deposition of the volatiles in icy form on the body surface could rejuvenate its appearance with bluish (or less red) colors.

\subsection{Sub-populations among the classicals.}

Tegler \& Romanishin (2000) found first indications that classical objects in near-circular orbits beyond 40 AU have very red colors and might thus constitute a separate group of objects in the Kuiper Belt. Other authors considered the existence of two separate groups as a function of orbital inclination (Brown 2001; Levison \& Stern 2001; Doressoundiram et al. 2002). The most reliable results about the existence of the two groups are shown in Peixinho et al. (2004). Based on their color-inclination properties and using a squared ranks test (Talwar \& Gentle 1977; Conover \& Iman 1978) Peixinho et al. obtained two distinct populations within the classical TNOs: the dynamically hot population $\left(i>4.5^{\circ}\right)$ which show low reddening values and a cluster of dynamically cold TNOs $\left(i<4.5^{\circ}\right)$ with very red colors. Hence, in the following we consider the possible correlations separately for the two populations, applying the selection criterium by inclination.

\subsubsection{Dynamically hot classicals $(n=42)$}

The dynamically hot classicals are considered those with inclination $i>4.5^{\circ}$. The total sample $(\mathrm{OWN}+\mathrm{LP}+2 \mathrm{MS})$ contains 42 objects with colors measured of which our new observations contribute $24 \%$.

Strong correlations: the main results obtained by other authors (Peixinho et al. 2004; Doressoundiram et al. 2005) are reproduced by us with minor differences. Particularly noteworthy are the strong correlations of colors versus $i$, and versus $q$ (Table 7) as for instance obtained also by Doressoundiram et al. (2005).

Weak correlations: a weak (anti-)correlation is found for $B-V$ vs. $e(\rho=-0.34, \mathrm{SL}=89.33 \%, n=23)$. The spectral gradient of this sample, based upon the $B-V$ color values, varies from -2.5 to $41.8 \% / 100 \mathrm{~nm}$ with an average value of $19.3 \pm$ $6.2 \% / 100 \mathrm{~nm}$. The anti-correlation found may indicate that the redder hot classicals have the lower eccentricity orbits.

Dependence on "sizes": we find the same trend for the dynamically hot population as for all classicals with regard to the color versus perihelion correlation (though with a slight increase in the SL when $\left.H_{R}<6.2(D>190 \mathrm{~km})\right)$, see Table 8 . Beyond that, we obtain a clear dependence on size for the color versus semimajor axis correlations (Table 8), in particular for $B-R$ vs. a $\left(H_{R}<6.2, D>190 \mathrm{~km}\right)$. This correlation is complemented by those for $V-R$ vs. $\psi$, Grt vs. a for $H_{R}<6.2$ (Tables 8, and 9) and $V-R$ vs. a for $H_{R}<6.2$ (Fig. 3, Table 8). Similar correlations are not found for the fainter objects $\left(H_{R}>6.2\right)$. Finally, we would like to mention that there might be a dependence on size for reddening gradients versus aphelion distance, as shown in Table 9 for Grt vs. $Q\left(H_{R}<6.2\right)$. Size dependence is not seen for the correlations of colors versus inclination and eccentricity, nor for spectral gradients.

Thus, considering all the size dependences for the dynamically hot classicals (versus $q, Q$ and $a$ ), we summarize that for the larger objects in this population $(D>190 \mathrm{~km})$ the redder ones have the larger perihelion \& aphelion distances, and semimajor axes. This is consistent with a resurfacing scenario by ice deposition due to a (temporal) bound coma as suggested by 


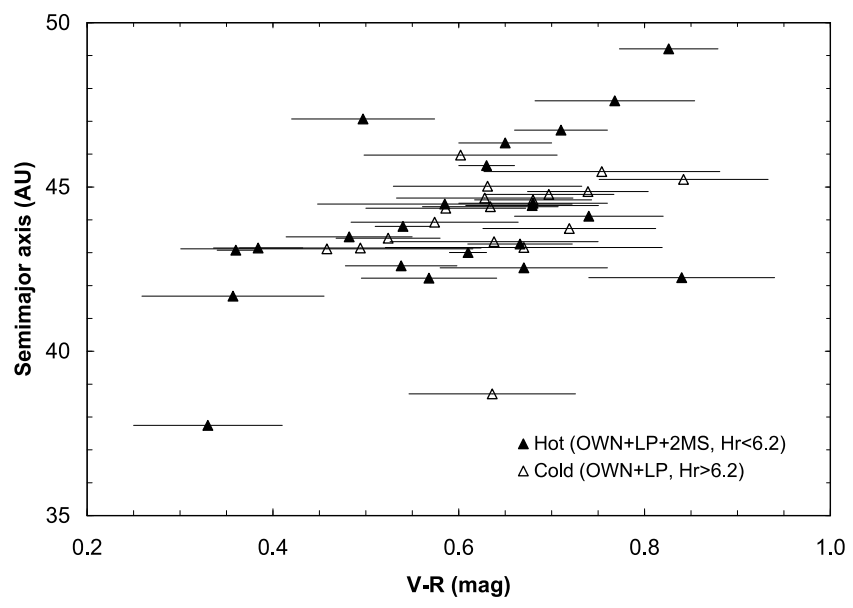

Fig. 3. $V-R$ vs. $a$ for $22(\mathrm{OWN}+\mathrm{LP}+2 \mathrm{MS})$ hot classical objects (filled triangles) with $H_{R}<6.2(D>190 \mathrm{~km})$ and for $17(\mathrm{OWN}+\mathrm{LP})$ cold classical objects (open triangles) with $H_{R}>6.2(D<190 \mathrm{~km})$.

Delsanti et al. (2004). From our results we may be actually seeing different behaviors for objects reflecting two different histories: resurfacing of objects with $D>190 \mathrm{~km}$ might be dominated by the accretional history of the body, while that of objects with $D<190 \mathrm{~km}$ might be dominated by their collisional history. However, our results are also compatible with the explanation scenario by Mc Kinnon (2002) for the blue coloring of the larger classical TNOs. He takes into account the internal evolution of the TNOs that are able to mobilize CO, e.g., through surface venting, and subsequent $\mathrm{CO}$-ice deposition on the exterior, cold, porous layers. This outgassing behaviour could be accelerated in objects with smaller perihelion distances. Also, the TNO resurfacing by meteoroid bombardment may play a greater role for smaller distances, as mentioned in Sect. 3.2. At the moment, we cannot distinguish the importance of the various proposed scenarios for the population of dynamically hot classical TNOs. However, the various correlations found between surface and dynamical properties of this group support the existence of a separate population of dynamically hot classicals that are distinctly different from the other TNOs.

\subsection{Dynamically cold classicals $(n=31)$}

This group of classicals $(n=31)$ consists of objects with orbital inclination $i<4.5^{\circ}$. Our new measurements of TNOs contribute $29 \%$ of the total sample (OWN+LP+2MS) of dynamically cold classicals with colors measured. New and known (confirmed) correlations of color versus orbital parameters are discussed below.

Strong correlations: we do not find strong correlations for the cold classicals objects (except a color-color one).

Weak correlations: a new weak correlation is found for color $R-I$ vs. $q(\rho=0.27, \mathrm{SL}=84.35 \%, n=29$; Table 8). The highest colors vs. orbital parameter correlation has been obtained for $V-R$ vs. $a$, which is a known weak correlation ( $\rho=$ $0.35, \mathrm{SL}=91.29 \%, n=25$; Table 8 ). However, we believe that color photometry of more cold classicals is required to confirm these results.

Dependence on "sizes": we find a stronger correlation for $R-I$ vs. $q(\rho=0.60, \mathrm{SL}=98.06 \%, n=16)$ for cold classicals with $H_{R}>6.2$, a result that is obtained for the whole classical group as well (Table 8). Since the hot classicals do not show such a dependence, we conclude it is characteristic of the cold population only. $V-R$ vs. $a$ correlates better for objects with $H_{R}>6.2$, and worse for those with $H_{R}<6.2$ (Fig. 3, Table 8), i.e. the behaviour of the dynamically cold classicals is opposite to that of the group of all classicals and in particular to that of the dynamically hot ones. Along with some distinct color-color correlations ( $B-V$ vs. $V-R$ and $V-R$ vs. $R-I$, on Table 7 ) that display opposite spectral slopes for these two classical groups, it could be indicative of a different primordial origin of the hot and cold classical populations. According to Morbidelli et al. (2003), the dynamically hot bluish population could have formed in a region closer to the Solar System than the dynamically cold reddish one. Neptune's migration is made responsible for shifting both populations to their current distance range in the Kuiper Belt. Based upon the correlations discussed above, one can speculate that a coloring mechanism may exist that acts in the opposite way and depending on $q$ and $a$ for the smaller cold classicals, compared to the hot ones.

\subsection{Scattered disk objects SDOs $(n=25)$}

The total sample of SDOs with colors measured comprises 25 objects. Our new measurements contribute $40 \%{ }^{2}$ to the total sample $(\mathrm{OWN}+\mathrm{LP}+2 \mathrm{MS})$. Our correlation analysis has led to results that are very similar to the ones obtained by other authors (Peixinho et al. 2004; Doressoundiram et al. 2005).

Strong correlations: besides correlations versus $q, H_{R}$, and $e$ as mentioned by Peixinho et al. (2004) and Doressoundiram et al. (2005), we find correlations for $B-V$ vs. $q, B-V$ vs. $H_{R}$, $B-V$ vs. $e$, and $R-I$ vs. $e$ (for the correlation characteristics see Table 7). Contrary to Doressoundiram et al. (2005) who claim a weak correlation for $B-R$ vs. $e$, we find an anti-correlation for $B-V$ vs. $e$. This finding (Fig. 4) is supported by the correlation results for Grt vs. $e$ (Table 9) and could mean that SDOs with lower eccentricities are redder than the higher eccentricity ones. In consequence, the SDOs in high eccentricity orbits may be more subject to a resurfacing mechanism that produces bluish colors. From the known correlations $B-R$ vs. $H_{R}$ displays the highest confidence level ( $\rho=-0.81, \mathrm{SL}=99.49 \%, n=13)$. Overall, the correlation results suggest that color vs. $H_{R}$ seem to play an important role in the SDOs group (Table 7). This is also seen for the respective Grt vs. $H_{R}$ correlations (see Table 9). We find a similar trend as found by Peixinho et al. (2004) for $B-R$ vs. $q$, although the significance level seems to decrease for larger sample sizes $(n=13: \rho=0.62, \mathrm{SL}=96.85 \% ; n=19: \rho=$ 0.34 , SL $=85.54 \%$; see Table 7 ).

Weak correlations: our analysis provides evidence for a weaker correlation between $B-V$ and $i$ that is known from previous datasets (Table 7). This correlation could imply that SDOs in higher inclination orbits are redder than those with lower inclination. Since the sample size is small $(n=13)$, additional SDO photometry would allow us to confirm or disprove the weak correlation mentioned above.

We found some additional weak correlations between colors and orbital elements as follows: $R-I$ vs. $q$ and $B-R$ vs. $e$.

Regarding $H_{R}$, inclination and eccentricity correlations we can say that the SDOs with fainter absolute magnitudes (i.e. the smaller ones), low inclinations, and high eccentricities appear typically bluer. Unfortunately, the abovementioned resurfacing scenarios are unable to account for these (weak) correlations and no simple mechanism is known that could produce such coloring profiles properly. So, one may have to think of combined effects,

2 Including the peculiar distant object $2000 \mathrm{CR}_{105}$. 


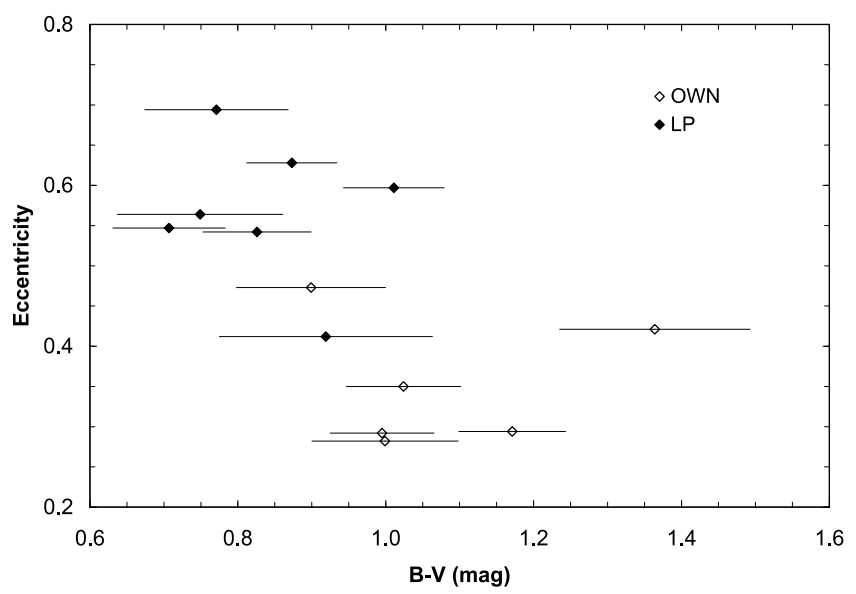

Fig. 4. $B-V$ vs. $e$ for 13 SDOs (OWN+LP). Symbols: OWN sample (open diamonds) and LP sample (filled diamonds).

and in any case reevaluate the respective correlation characteristics based upon a better and larger SDO sample.

\subsection{Combined groups}

In order to analyse possible color relationships among the various dynamical groups of TNOs we consider in the following two samples of combined population, e.g. SDOs with hot classicals and SDOs with Centaurs. The common property of the combined groups is that their orbits were subject to gravitational scattering in the past. Hence, by comparing the results of the combined samples with those of the individual ones described above, it may be possible to isolate possible similarities and diversities that may allow us to constrain common or different origin and/or evolution. For the analysis, one must take into account that in the combined sample, individual groups have different weights according to the respective sample sizes.

\subsubsection{Merged sample: SDOs+hot classicals $(n=67)$}

The total number of objects in this sample is 67 of which 42 are hot classicals and 25 are SDOs.

Some correlation evidence in the merged sample increases with respect to the SDOs alone, for instance $V-R$ vs. $i$, and $B-R$ vs. $i$. There are also some that disappear, for instance $B-V$ vs. $H_{R}, B-V$ vs. $e, V-R$ vs. $H_{R}, R-I$ vs. $q, R-I$ vs. $e$, $B-R$ vs. $H_{R}$, and $B-R$ vs. $e$. The increase in the color vs. $i$ correlations is clearly due to the hot classicals in the merged sample (Table 7). For the comparison between the merged sample and hot classicals we again have a similar situation. Here, we would like to mention in particular the less prominent or vanishing correlations, e.g. $B-V$ vs. $i, B-V$ vs. $e, V-R$ vs. $a, B-R$ vs. $q$, $B-R$ vs. $i, B-R$ vs. $e$, and $B-R$ vs. $a$ (Table 7 ).

In summary, there is no significant strengthening for colororbit correlations seen in the merged group of SDOs and hot classicals such that we can argue for a common origin or very similar evolution.

\subsubsection{Merged sample: SDOs+Centaurs $(n=43)$}

The merged sample of 43 objects consists of 25 SDOs and 18 Centaurs.
The situation for the SDOs+Centaurs sample compared to SDOs and Centaurs alone is again unchanged, with a few moderately stronger correlations compared to SDOs alone on one side, (for instance for $R-I$ vs. $i$, and $B-R$ vs. $a$ ) and weaker or disappearing correlations on the other side ( $B-V$ vs. $q, B-V$ vs. $H_{R}, B-V$ vs. $i, B-V$ vs. $e, V-R$ vs. $q, V-R$ vs. $H_{R}, R-I$ vs. $q, R-I$ vs. $e, B-R$ vs. $q, B-R$ vs. $H_{R}$, and $B-R$ vs. $e$ ). The increase of the correlation evidence in color vs. $i$ and $a$ is possibly $a$ contribution from the Centaur group. Compared to the Centaurs alone we find increased evidence for correlations between $R-I$ and $e, B-R$ and $q$, and $B-R$ and $H_{R}$, certainly due to the SDO contribution in the merged sample. On the other side decreased or disappearing evidence is found in the merged sample for the correlations $B-V$ vs. $i, B-V$ vs. $e, V-R$ vs. $q$, $V-R$ vs. $H_{R}, V-R$ vs. $e, V-R$ vs. $a, R-I$ vs. $H_{R}, R-I$ vs. $i$, $B-R$ vs. $i, B-R$ vs. $e$, and $B-R$ vs. $a$.

In summary, as for the other merged sample, no clear changes in the correlation significances are identified that would allow us to argue for or against a possible common origin and/or evolution of the SDOs and Centaurs.

\section{Conclusions}

- BVRI photometry results on 32 KBOs with ESO/VLT telescope are presented (colors and absolute magnitudes). Merging our photometric results with the ESO and 2MS data sets, we obtain a large and rather homogeneous sample of 116 objects that is used for statistical analysis of correlations between colors and/or orbital parameters of the objects.

- We found short-term brightness variability due to rotation in 11 of the 32 objects studied. While the percentage of objects with very high amplitudes is similar to results published before, our sample has a higher fraction of objects with medium to small amplitude variations. Since our sample includes objects with fainter magnitudes (i.e. smaller sizes) than previous studies, we tentatively conclude that smaller objects have, on average, more irregular shapes than larger ones. Independent confirmation of this conclusion with a much larger sample is highly desirable.

- Our average spectral gradient calculations are compatible with previous results:

$\mathrm{Grt}_{\text {avg }}($ Class, $n=73)=22.9 \pm 9.8 \% / 100 \mathrm{~nm}$

$\mathrm{Grt}_{\mathrm{avg}}($ Hot, $n=42)=19.6 \pm 8.5 \% / 100 \mathrm{~nm}$

$\mathrm{Grt}_{\mathrm{avg}}($ Cold, $n=31)=27.4 \pm 11.3 \% / 100 \mathrm{~nm}$

$\mathrm{Grt}_{\mathrm{avg}}(\mathrm{SDOs}, n=25)=18.6 \pm 7.6 \% / 100 \mathrm{~nm}$

$\mathrm{Grt}_{\mathrm{avg}}($ Cent, $n=18)=19.8 \pm 6.7 \% / 100 \mathrm{~nm}$.

- We obtain color vs. perihelion distance correlations, and color vs. inclination and eccentricity anticorrelations for the classical TNOs. These results imply that the redder classical objects have low inclinations and eccentricities, and high perihelion distances. This interpretation supports the existence of a cold red cluster of classical objects in very circular orbits with low inclinations, and possibly supports an ice resurfacing scenario that depends on the solar distance.

- A size dependence of the correlation of colors versus semimajor axis is found in classical objects. We use an absolute magnitude cuttoff value of $H_{R}=6.2$. For the large objects $\left(H_{R}<6.2, D>190 \mathrm{~km}\right.$, assuming $\left.p_{R}=0.12\right)$ the redder ones have the larger semimajor axes. This result could support a possible resurfacing mechanism depending on semimajor axis. The large objects sizes might allow them to develop a bound coma, and perhaps a coloring mechanism due to sublimation, and recondensation can take place. No apparent dependence on sizes of the correlations of colors versus 
perihelion distances, inclination or eccentricity are found for classical objects.

- We find in the hot $\left(i>4.5^{\circ}\right)$ classical objects that the redder ones have the larger perihelion distances, and the lower orbital inclinations. We find that correlations of color versus perihelion distance, aphelion distance and semimajor axis depend on size: these correlations increase for the larger $(D>190 \mathrm{~km})$ hot classical objects. These results support a coloring mechanism that depends on solar distance and size.

- Two possible color versus semimajor axis, and perihelion distance correlations are found for the cold $\left(i<4.5^{\circ}\right)$ group. These results seem to have a relevant dependence on sizes: the smaller $(D<190 \mathrm{~km})$ cold classical objects correlate better with perihelion and semimajor axis than the bigger ones. In other words, the smaller redder cold objects would have higher semimajor axe and perihelion distances. So, it seems there is a solar distance dependent coloring mechanism different to ice resurfacing from sublimation and redeposition for the smaller cold objects (the small mass does not permit the development of a bound coma).

- A possible different primordial origin for the hot and cold populations is consistent with our correlations and statistical analysis. We find seven higher correlations for the hot group than for the classical one. The $V-R$ vs. $a$ correlation presents an opposite trend in size for the hot and cold groups: for the hot objects the $V-R$ vs. semimajor axis increases for the larger ones $\left(H_{R}<6.2, D>190 \mathrm{~km}\right)$, and for the cold objects it increases for the smaller ones $\left(H_{R}>6.2, D<190 \mathrm{~km}\right)$.

- The SDOs with higher absolute magnitudes (i.e. the smaller ones), lower inclinations and perihelion distances, and higher eccentricities are the bluer ones. A possible physical process that may produce such a coloring scheme is currently unknown to us.

- The Centaurs with higher inclinations, lower eccentricities, smaller sizes and lower semimajor axis are the bluer ones. This result is opposite to that obtained for the SDOs, so the coloring mechanism for Centaurs and SDOs must be different.

- We do not find relevant differences between the statistical results for merged groups (i.e. SDOs+hot, SDOs+Cent), so this cannot be used to link different dynamical groups or to say something about a possible common origin and/or evolutionary relation between these different dynamical groups.

Acknowledgements. We are grateful to the Cerro Paranal Observatory staff. This research was based on data obtained at the VLT on Cerro Paranal Observatory, which is operated by the European Southern Observatory (ESO). This work was supported by contracts AYA-2002-00382, AYA-2004-03250, and AYA-200806202-C03-01. P.S.S. wishes to acknowledge J. D. Santander-Vela for his help with the manuscript.

\section{References}

Belskaya, I. N., Ortiz, J. L., Rousselot, P., et al. 2006, Icarus, 184, 277 Boehnhardt, H., Tozzi, G. P., Birkle, K., et al. 2001, A\&A, 378, 653 Boehnhardt, H., Delsanti, A., Barucci, A., et al. 2002, A\&A, 395, 297 Boehnhardt, H., Barucci, A., Delsanti, A., et al. 2003, EM\&P, 92, 145 Bowell, E., \& Lumme, K. 1979, Asteroids, 132

Bowell, E., Hapke, B., Domingue, D., et al. 1989, in Asteroids II (University of Arizona Press), 524

Brown, M. E. 2001, AJ, 121, 2804

Brown, M. E., \& Trujillo, C. A. 2004, AJ, 127, 2413

Conover, W. J., \& Iman, R. L. 1978, Commun. Stat. B, 7, 491

Cruikshank, D. P., Stansberry, J. A., Emery, J. P., et al. 2005, ApJ, 624, L53

Davies, J. K., Green, S., McBride, N., et al. 2000, Icarus, 146, 253

Delsanti, A., Hainaut, O., Jourdeuil, E., et al. 2004, A\&A, 417, 1145

Delsanti, A., Peixinho, N., Boehnhardt, H., et al. 2006, AJ, 131, 1851

Delsemme, A. H. 1982, in Comets (Tucson, AZ: University of Arizona Press), 85

Doressoundiram, A., Peixinho, N., de Bergh, C., et al. 2002, AJ, 124, 2279

Doressoundiram, A., Peixinho, N., Doucet, C., et al. 2005, Icarus, 174, 90

Duncan, M., Quinn, T., \& Tremaine, S. 1988, ApJ, 328, L69

Duncan, M. J., Levison, H. F., \& Budd, S. M. 1995, AJ, 110, 3073

Efron, B., \& Tibshirani, R. J. 1993, An Introduction to the Bootstrap (Chapman \& Hall/CRC)

Fernández, J. A. 1980, MNRAS, 192, 481

Fornasier, S., Doressoundiram, A., Tozzi, G. P., et al. 2004, A\&A, 421, 353

Gladman, B., Holman, M., Grav, T., et al. 2002, Icarus, 157, 269

Gomes, R. S. 2003, Icarus, 161, 408

Grundy, W. M., Noll, K. S., \& Stephens, D. C. 2005, Icarus, 176, 184

Hainaut, O. R., \& Delsanti, A. C. 2002, A\&A, 389, 641

Hainaut, O. R., Delahodde, C. E., Boehnhardt, H., et al. 2000, A\&A, 356, 1076

Howell, S. B. 1989, PASP, 101, 616

Jewitt, D. C., \& Meech, K. J. 1986, AJ, 310, 937

Jewitt, D., \& Luu, J. 1993, Nature, 362, 730

Jewitt, D., Aussel, H., \& Evans, A. 2001, Nature, 411, 446

Lacerda, P., \& Luu, J. 2006, AJ, 131, 2314

Levison, H. F., \& Stern, S. A. 2001, AJ, 121, 1730

Lykawka, P. S., \& Mukai, T. 2005, P\&SS, 53, 1319

Margot, J. L., Trujillo, C., Brown, M. E., \& Bertoldi, F. 2002, BAAS, 34, 871

McKinnon, W. B. 2002, Asteroids, Comets, and Meteors: ACM 2002, 500, 29

Millis, R. L., Bowell, E., \& Thompson, D. T. 1976, Icarus, 28, 53

Morbidelli, A., Brown, M. E., \& Levison, H. F. 2003, EM\&P, 92, 1

Noll, K. S., Stephens, D. C., Grundy, W. M., \& Griffin, I. 2004, Icarus, 172, 402

Opik, E. J. 1976., in Interplanetary Encounters (Amsterdam: Elsevier), 27

Ortiz, J. L., Gutierrez, P. J., Santos Sanz, P., Casanova, V., \& Sota, A. 2006, A\&A, 447, 1131

Osip, D. J., Kern, S. D., \& Elliot, J. L. 2003, EM\&P, 92, 409

Peixinho, N., Boehnhardt, H., Belskaya, I., et al. 2004, Icarus, 170, 153

Rabinowitz, D. L., Schaefer, B. E., \& Tourtellotte, S. W. 2007, AJ, 133, 26

Sheppard, A., \& Jewitt, D. 2002, AJ, 124, 1757

Sheppard, A., \& Jewitt, D. 2003, EM\&P, 92, 207

Spearman, C. 1904, The proof and measurement of association between two things, Am. J. Psychol., 57, 72

Stansberry, J. A., Cruikshank, D. P., Grundy, W. G., et al. 2005, BAAS, 37, 737

Stansberry, J., Grundy, W., Brown, M., et al. 2008, in The Solar System Beyond Neptune (U. Arizona Press), 161

Talwar, P. P., \& Gentle, J. E. 1977, Commun. Stat.-Theory, 6, 363

Tegler, S. C., \& Romanishin, W. 2000, Nature, 407, 979

Tegler, S. C., Romanishin, W., \& Consolmagno, G. J. 2003, ApJ, 599, L49

Thébault, P. 2003, EM\&P, 92, 233

Thébault, P., \& Doressoundiram, A. 2003, Icarus, 162, 27

Trujillo, C. A., \& Brown, M. E. 2002, ApJ, 566, L125 